ARTICLE

\title{
In vivo CRISPR/Cas9 targeting of fusion oncogenes for selective elimination of cancer cells
}

\author{
M. Martinez-Lage (10) ${ }^{1,10}$, R. Torres-Ruiz (10 1,2,10凶 , P. Puig-Serra (1) 1, P. Moreno-Gaona ${ }^{1}$, M. C. Martin ${ }^{1}$, F. J. Moya ${ }^{1}$, \\ O. Quintana-Bustamante (10) 3,4, S. Garcia-Silva (1) ${ }^{5}$, A. M. Carcaboso (1) 6,7, P. Petazzi ${ }^{2}$, C. Bueno ${ }^{2}$, J. Mora (i) ${ }^{6,7}$, \\ H. Peinado (i) 5 , J. C. Segovia (i] ${ }^{3,4}$, P. Menendez ${ }^{2,8,9} \&$ S. Rodriguez-Perales (1) ${ }^{1 \times}$
}

Fusion oncogenes (FOs) are common in many cancer types and are powerful drivers of tumor development. Because their expression is exclusive to cancer cells and their elimination induces cell apoptosis in FO-driven cancers, FOs are attractive therapeutic targets. However, specifically targeting the resulting chimeric products is challenging. Based on CRISPR/Cas 9 technology, here we devise a simple, efficient and non-patient-specific gene-editing strategy through targeting of two introns of the genes involved in the rearrangement, allowing for robust disruption of the FO specifically in cancer cells. As a proof-of-concept of its potential, we demonstrate the efficacy of intron-based targeting of transcription factors or tyrosine kinase FOs in reducing tumor burden/mortality in in vivo models. The FO targeting approach presented here might open new horizons for the selective elimination of cancer cells.

\footnotetext{
${ }^{1}$ Molecular Cytogenetics and Genome Editing Unit, Human Cancer Genetics Program, Centro Nacional de Investigaciones Oncológicas (CNIO), 28029 Madrid, Spain. ${ }^{2}$ Josep Carreras Leukemia Research Institute and Department of Biomedicine, School of Medicine, University of Barcelona, 08036 Barcelona, Spain. ${ }^{3}$ Differentiation and Cytometry Unit, Hematopoietic Innovative Therapies Division, Centro de Investigaciones Energéticas, Medioambientales y Tecnológicas (CIEMAT), Centro de Investigaciones Biomédicas en Red de Enfermedades Raras (CIBERER), 28040 Madrid, Spain.

${ }^{4}$ Advanced Therapies Mixed Unit, Instituto de Investigación Sanitaria-Fundación Jiménez Díaz (IIS-FJD, UAM), 28040 Madrid, Spain. ${ }^{5}$ Microenvironment and Metastasis Group, Molecular Oncology Program, Spanish National Cancer Research Centre, 28029 Madrid, Spain. ${ }^{6}$ Institut de Recerca Sant Joan de Deu, Barcelona, Spain. ${ }^{7}$ Department of Pediatric Hematology and Oncology, Hospital Sant Joan de Deu, 08950 Barcelona, Spain. ${ }^{8}$ Instituciò Catalana de Recerca i Estudis Avançats (ICREA), Passeig Lluis Companys, 08010 Barcelona, Spain. ${ }^{9}$ Centro de Investigación Biomédica en Red de Cáncer (CIBER-ONC), ISCIII, Barcelona, Spain. ${ }^{10}$ These authors contributed equally: M. Martinez-Lage and R. Torres-Ruiz. ${ }^{凶}$ email: rtorresr@cnio.es; srodriguezp@cnio.es
} 
D espite advances in treatment, cancer continues to cause over nine million deaths worldwide each year. Indeed, there is still much to be learned about the biologic behavior of cancer cells and molecular disease mechanisms. In this sense, genome editing offers unprecedented opportunities to further our knowledge of cancer biology and to fuel the development of new preclinical models and more efficient and directed strategies to eliminate cancer cells ${ }^{1}$. Unlike other genetic diseases such as Duchenne muscular dystrophy or cystic fibrosis, cancer development involves several genetic mutations that can deregulate multiple genes. In the context of cancer gene therapy, it is clear that targeting a single gene is often insufficient to eliminate cancer cells-yet, many types of cancers are addicted to the presence of a single oncogenic event that can reprogram cells by deregulating downstream molecular and (epi)-genetic programs ${ }^{2}$ and initiate tumorigenesis. This is the case for the so-called fusion oncogenes (FOs), which are chimeric genes resulting from inframe fusions of the coding sequences of two genes involved in a chromosomal rearrangement ${ }^{3}$. While the nature of the FOs may be diverse, they are primarily classified as involving transcription factors or tyrosine kinases. Silencing of FO transcripts has been shown to inhibit tumor cell growth in vitro and in vivo ${ }^{4,5}$, demonstrating FO addiction in many human cancers. Moreover, genome-wide mutational studies-particularly in childhood cancers-have provided additional support for some FOs as the sole necessary genetic drivers for cancer initiation by revealing silent mutational landscapes in FO-initiated tumors 6,7 .

FOs are recurrent genomic findings in human cancer and are characterized by patient-specific genomic breakpoints that occur in intronic regions, rarely disrupting coding sequences. Analysis of data from The Cancer Genome Atlas suggests that FOs drive the development of more than $16 \%$ of human cancers $^{8}$, including mesodermal cancers (typically leukemias, lymphomas and sarcomas). FOs have also been found in epithelial cancers, including prostate ${ }^{9}$, colorectal $^{10}$, breast ${ }^{11}$ or melanoma ${ }^{12}$, and over 350 recurrent FOs involving $>300$ different genes have been identified to date ${ }^{13,14}$. Given their restriction to cancer cells, FOs are attractive targets for directed therapy; however, therapeutic targeting of specific FOs has remained challenging due to the difficulties in specifically recognizing and targeting the resultant chimeric protein, and also because FO products are intracellular, necessitating effective intracellular approaches for delivery of therapeutic molecules targeting the chimeric transcripts/proteins ${ }^{15}$. Along this line, small molecules ${ }^{16}$, intrabodies ${ }^{17}$ and aptamers ${ }^{18}$ have been used successfully to target fusion proteins, and antisense RNA $^{19}$, ribozymes ${ }^{20}$ and $\mathrm{RNAi}^{21}$ to target the fusion transcripts. Likewise, the development of genome editing approaches offers new possibilities to directly target and modify the genomic sequence of cancer cells ${ }^{22}$.

Clustered Regularly Interspaced Short Palindromic Repeats (CRISPR)/Cas9-based technology has revolutionized genome editing of mammalian cells, and can generate targeted breaks at any desired location in the genome-opening new horizons for therapeutic gene editing to correct monogenic or somatic mutations ${ }^{23,24}$. CRISPR/Cas9 induced breaks can be repaired by one of two major double-strand break (DSB) repair pathways: the inefficient but error-free homology-directed repair (HDR) pathway, which requires a DNA template; and the highly efficient but error-prone non-homologous end joining (NHEJ) pathway, which does not require a DNA template. A recent pioneering study by Chen et al. exploited the HDR repair pathway to insert a suicide gene at patient-specific breakpoints of FOs in prostate and hepatocellular cancer cell lines ${ }^{25}$. Although very elegant, the combination of an extremely low efficiency (1-10\%) HDR-based approach $^{26}$ and the requirement of patient-specific breakpoint
CRISPR tool development limits the utility of this strategy for developing targeted cancer therapies.

In the present study, we report an efficient NHEJ CRISPRmediated genome editing strategy for targeting FOs, which we believe represents a valid approach for directed elimination of cancer cells harboring a given FO. The approach is based on targeting two intronic sequences-one in each of the genes involved in the FO-that induces a cancer cell-specific genome deletion that eliminates key protein domains or changes the reading frame of the FO. Notably, this gene editing-based approach induces deletion only in cells harboring a FO without affecting exonic sequences or protein expression of the germline non-rearranged alleles. The same two single guide RNAs (sgRNAs) allow the targeting of different isoforms or every patient-specific breakpoint of a given FO, and is thus a universal approach for cancer-associated FOs. In vitro analysis and patientderived xenograft (PDX) models show that delivery of CRISPR/ Cas9 components targeting a FO results in efficient and specific tumor growth control. Our findings provide a proof-of-concept for a highly efficient and non-breakpoint-specific genome editing strategy-targeting FOs as an innovative approach for selective elimination of cancer cells.

\section{Results}

CRISPR-mediated intronic targeting enables FO disruption. Our rationale was to devise a genome editing approach to specifically disrupt FOs in cancer cells that fulfils two strict criteria: i) it would not affect the exonic sequences or the expression of wildtype alleles involved in the rearrangement, and ii) it would be feasible irrespective of the FO isoform or the patient-specific breakpoint. To test this approach, we first used a cellular model of Ewing sarcoma, one of the most common cancers in children/ adolescents characterized by a chromosomal translocation that fuses the transactivation domain of the RNA-binding protein EWSR1 to the DNA-binding domain of an ETS protein, most commonly FLI1. The EWSR1-FLI1 (EF) fusion acts as a dominant transcription factor, and cells are addicted to EF expression ${ }^{27-29}$. Two main $E F$ isoforms exist that fuse exon 7 of EWSR1 to either exon 5 (EF type 2 ) or exon 6 of FLI1 (EF type 1$)^{30-32}$.

We designed a strategy to induce $E F$-specific genomic deletion targeting two genomic introns-one in each rearranged gene flanking the breakpoint introns. To target all isoforms of $E F$, four sgRNAs were designed to target introns 3 and 6 of EWSR 1 and introns 6 and 8 of FLI1 (Fig. 1a and Supplementary Table 1). sgRNAs were designed specifically to not disrupt described splice acceptor or donor sites or transcription regulators such as enhancers or silencers. We also confirmed that the sgRNA target sites were not affected by common single nucleotide polymorphisms (SNPs) (Supplementary Fig. 1a, b). Targeted introns were selected to generate large deletions including key functional domains of the FO, to induce a frameshift event in the remaining $3^{\prime}$ region of the FLI1 gene, and to cover all the common hotspot introns within the break cluster regions. Consequently, genomic deletions will occur only in cells harboring the FO with both ontarget intronic regions in the same chromosome. Crucially, intron-directed sgRNAs guarantee the germline configuration of non-rearranged EWSR1 and FLI1 alleles, such that the expression of wild-type alleles is preserved in healthy cells.

Using a single sgRNA lentiviral expression vector ( $\mathrm{pLV}$ U6sgRNA-EFSCas9) ${ }^{33}$, we tested the efficiency of genomic deletion with four combinations of sgRNAs (sgE3-sgF6, sgE3sgF8, sgE6-sgF6, sgE6-sgF8) in the A673 Ewing sarcoma cell line. Sanger sequencing analysis of PCR products using oligonucleotides flanking the targeted loci confirmed genomic deletions (Supplementary Fig. 2a), and EF-targeted A673 cells showed a 
a

\section{EWSR1-FLI1}

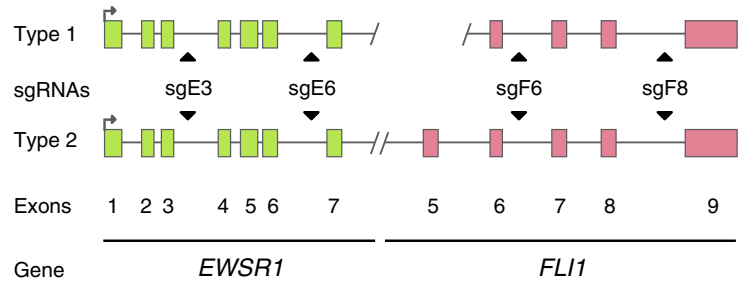

b

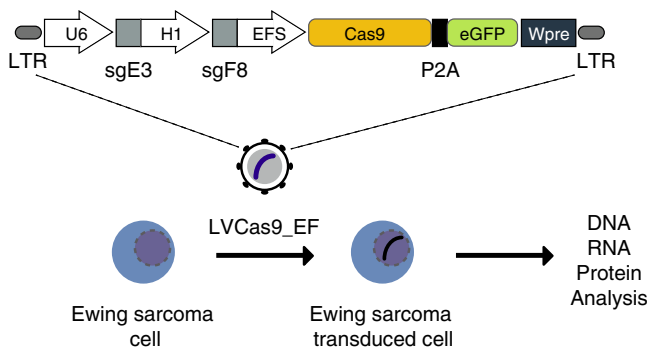

e

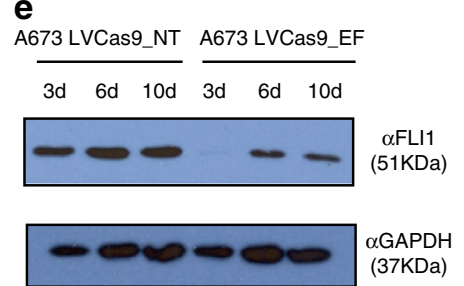
(37KDa)

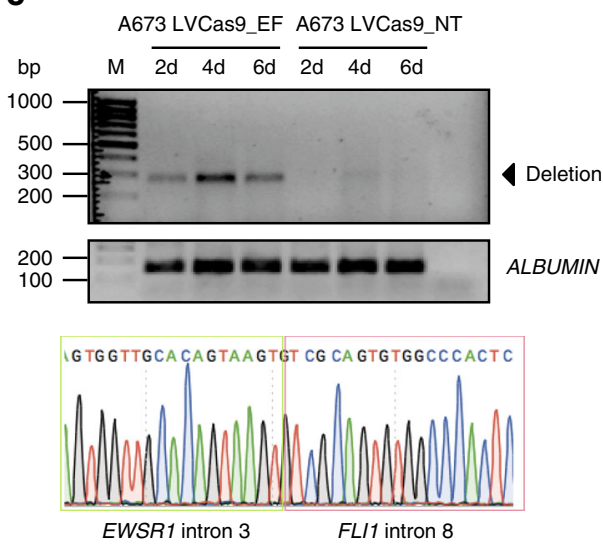

d

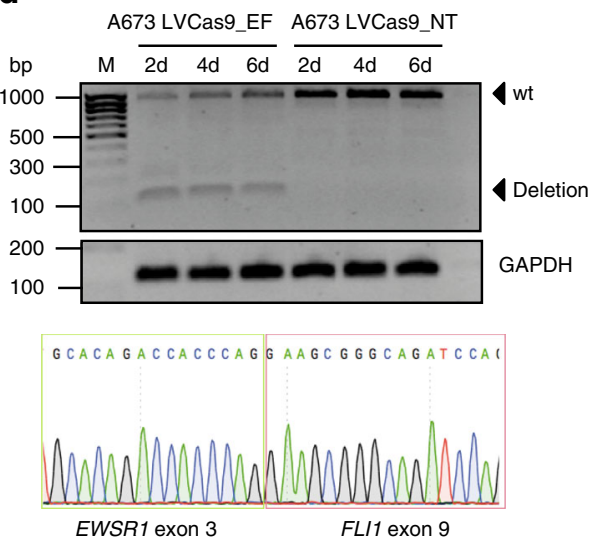

Fig. 1 Strategy and in vitro CRISPR-mediated disruption of EWSR1-FLI1. a Scheme representing type 1 and 2 EWSR1-FLI1 FOs, illustrating the genomic structure with exon arrangement and sites of fusion. sgRNAs targeting introns 3 and 6 of EWSR1 and 6 and 8 of FLI1 are indicated. b Schematic representation of the all-in-one lentiviral vector for simultaneous expression of two sgRNAs, Cas 9 and eGFP regulated by the U6, H1, and EFS promoters. c, Genomic PCR analysis of edited and control A673 cells using oligonucleotides flanking the DNA targeted by sgE3 and sgF8 ( $n=3$, independent studies). The $300 \mathrm{bp}$ PCR product denotes deletion of the DNA fragment between the loci targeted by sgE3 and sgF8. PCR analysis was performed on extracted DNA of cells at day 2,4 , and 6 post-transduction (pt). ALBUMIN was used as an internal control of the PCR reaction. Bottom panel shows a representative Sanger sequencing chromatogram of the PCR products. d RT-PCR products from edited and control A673 Ewing sarcoma cells ( $n=3$, independent studies). Analysis was done using extracted RNA of cells at day 2, 4, and 6 pt. Arrows depict the sizes of wild-type (961 bp) and deleted (150 bp) RT-PCR products. GAPDH was used as an internal control of the RT-PCR reaction. Bottom panel shows a representative Sanger sequencing chromatogram of RTPCR products. e Western blotting of EWSR1-FLI1 in A673 cells. Analysis was done using total protein extracted from cells at day 3, 6, and 10 posttransfection using an antibody specific for FLI1. GAPDH was used as an internal control of the assay. LTR: Long term repeat; P2A: porcine teschovirus-1 2A self-cleaving peptide; WPRE: Virus (WHP) posttranscriptional regulatory elements.

significantly blunted clonogenic capacity $(51 \%, 62 \%, 49 \%$, and $56 \%$, respectively) irrespective of the sgRNA pair used (Supplementary Fig. 2b). Cleavage with sgE3-sgF8 resulted in high deletion efficiency, generating the largest $(27.7 \mathrm{~kb}) \mathrm{EF}$ deletion and resulting in the complete loss of the EWSR1 transactivation domain and a frameshift alteration of the entire FLI1 DNAbinding region (Supplementary Fig. 3a). Accordingly, this combination was chosen for further study. Notably, targeted deep-sequencing of sgE3- or sgF8-targeted A673 cells revealed $62 \%$ and $66 \%$ insertion/deletion (indels) in EWSR1 and FLI1 ontarget sites, respectively (Supplementary Table 2a, b). For subsequent targeting of $\mathrm{EF}, \mathrm{sgE} 3$ and $\mathrm{sgF} 8$ were cloned into an all-in-one expression plasmid ${ }^{34}$ (pLV-U6sgE3-H1sgF8-EFSCas92A-eGFP; hereafter termed LVCas9_EF) expressing sgE3 and sgF8 from the U6 and H1 RNApol III promoters, respectively, together with the simultaneous expression of Cas9 and GFP proteins separated by a $2 \mathrm{~A}$ self-cleaving peptide (Fig. 1b).

CRISPR-mediated deletion selectively reduces FO products. We first examined the capacity of LVCas9_EF to generate EF deletions in A673 and also RD-ES Ewing sarcoma cells, which harbor different EF isoforms. The pLV-U6sgNT-EFSCas9) ${ }^{33}$ single non-targeting vector (hereafter termed LVCas9_NT) was used as a control (Supplementary Table 1). PCR analysis of the genomic regions spanning the intronic cleavage sites
(Supplementary Table 1) revealed a PCR band verified by Sanger sequencing both in A673 cells (Fig. 1c) and in RD-ES cells (Supplementary Fig. 2c). Consistently, RT-PCR and western blot analysis confirmed that the simultaneous expression of sgE3 and sgF8 induced a robust loss of EF mRNA and protein, respectively (Fig. 1d, e and Supplementary Fig. 2d). We designed a quantitative qRT-PCR-based expression assay for FO to estimate rates of deletion, and found a significant 4.5-fold decrease in FO expression in edited A673 cells at day 3 post-transduction (p.t.) (Supplementary Fig. 2e). Deep sequencing of the deletion PCR amplicons indicated a preference $(\sim 60 \%)$ for precise ligation of cut products (Supplementary Fig. 2f). Finally, targeted deepsequencing analysis revealed that EF-targeted human primary mesenchymal stem cells (hMSC) cells perform accurate splicing of EWSR1 exons. To rule out any possible bias in the use of a non-targeting control, we also tested a second control plasmid (LVCas9_dC1/2) targeting two unrelated genomic loci that generate two genomic DSB in two different chromosomes. Results showed no changes to cell viability in transduced A673 cells (Supplementary Table 1 and Supplementary Fig. 2g).

CRISPR FO targeting does not impair genomic stability. We next evaluated whether our CRISPR-mediated EF targeting strategy induces collateral genomic alterations. We used as wildtype control clinically-relevant healthy hMSC, which are 
considered the cell-of-origin for human sarcomas ${ }^{35}$. Sequencing analysis of EF-targeted hMSCs confirmed the presence of a repertoire of indels ( $55 \%$ and $68 \%, E W S R 1$ and FLI1, respectively) at the targeted regions (Supplementary Fig. 4a). To assess possible side-effects that could affect proliferation of healthy cells, we treated hMSCs with LVCas9_NT or LVCas9_EF, finding no difference in proliferation rates in long-term culture (Supplementary Fig. 4b). Also, hMSC transduced with LVCas9_EF did not produce tumors when injected into immunosuppressed mice. G-banding karyotype analysis 7 days after transduction revealed no numerical or structural abnormalities in hMSC, irrespective of the vector used (Supplementary Fig. 4c), and high-density genome-wide array comparative genomic hybridization (aCGH) analysis confirmed no large copy number deletions across the genome (Supplementary Fig. 4d). hMSCs were also negative for EWSR1-FLI1 translocation products by FISH and PCR analysis at 15- and 30-days p.t. (Supplementary Fig. 4e, f and Table 1). Finally, qRT-PCR analysis of edited hMSCs confirmed nonsignificant variation in the levels of EWSR1 and FLI1 expression (Supplementary Fig. 4g). To test for potential off-target cleavage activity, we performed next-generation sequencing (NGS) of PCR amplicons spanning the 50 more probable in silico-predicted offtarget sites of $\mathrm{sgE} 3$ and $\mathrm{sgF}$. The threshold percentage for offtarget activity was set at $0.10 \%$, since this was the highest percentage of indel-containing reads in control samples, considering as noise indel-containing reads below this percentage ${ }^{36}$. For every off-target site analyzed, indel-containing reads represented less than $0.10 \%$ of the total, with the exception of one predicted site (Supplementary Table 3, Supplementary Fig. 4h). Although this site showed indel-containing reads at a slightly higher frequency $(\sim 0.12 \%)$ than the threshold, the sequence variations found were also present in both controls and edited samples, suggesting that they were not bona fide Cas9 off-targets. Thus, targeted NGS ruled out mutations in EF-targeted hMSCs, A673, and RD-ES cells harvested 7 days after LVCas9_EF transduction. Overall, these data show that the therapeutic targeting of the EF FO seems highly specific and does not interfere with non-rearranged alleles in human control healthy cells.

Targeting of a driver FO inhibits tumor cell growth in vitro. We utilized in vitro assays to examine the functional consequences of targeting EF. Transduction with LVCas9_EF, but not with LVCas9_NT, resulted in a $65.5 \%$ and $93.9 \%$ decrease in A673 and RD-ES cell growth, respectively (Fig. 2a, b), and a $60.5 \%$ and $70.3 \%$ decrease, respectively, in clonogenic capacity (Fig. 2d, e). No changes were found in LVCas9_EF-transduced control U2OS cells (non-EWSR1-FLI1) (Fig. 2c, f). Consistent with the growth phenotype observations, $E F$-targeted cells had higher levels (5-8-fold) of apoptosis, measured both by propidium iodide staining (subG1 cells, Fig. $2 \mathrm{~g}$ ) and caspase-3 analysis (Fig. $2 \mathrm{~h}$ ), than control transduced cells, suggesting that CRISPR-mediated abrogation of EF is sufficient to inhibit the survival, proliferation, and clonogenicity of $E F$-expressing Ewing sarcoma cells in vitro. As the induction of two DSBs in the same chromosome has been related to inversion events ${ }^{37}$, we checked for the presence of this type of rearrangement in A673 cells. PCR analysis revealed the presence of inversion products at 3 days p.t.; however, inversion carrier cells were lost during cell culture and no inversion products were detectable at day 21 p.t. (Supplementary Fig. 2h). In silico analysis predicted that an inversion of the FO targeted region will produce a truncated protein similar to that induced by DNA deletion (Supplementary Fig. 3a), which is compatible with a similar mechanism of cell death of inversion carrier cells.
FO deletion controls tumor growth in a xenograft model. We chose adenovirus (AdV) vectors to test the in vivo applications of the targeting approach because of their high gene transfer efficiency, their ability to infect cancer cells, their capacity for large DNA transgenes (allowing co-delivery of sgRNAs and Cas9 within single vector particles) and also because they do not readily integrate into host genomes ${ }^{38}$. To test whether AdV-based in vivo delivery of the FO-targeting CRISPR machinery can control tumor growth, we subcutaneously injected A673 cells into immunodeficient mice and allowed tumors to develop for 10 days ( $\sim 150 \mathrm{~mm}^{3}$ in size) (Fig. 3a). Tumors were then injected with 100 $\mu \mathrm{L}$ of PBS containing $2.5 \times 10^{9}$ plaque-forming units ( $\mathrm{pfu}$ ) of the AdCas9_EF vector containing the same cassette as described above for the LV vector (sgE3 and sgF8 together with Cas9 and GFP proteins), or the equivalent non-targeting AdCas9_NT control vector, or PBS, at day 10, 13, 16 and 19 (Fig. 3a). Results revealed that compared with AdCas9_NT- or PBS-treated mice, tumor size in AdCas9_EF-treated mice was reduced by $\sim 70 \%$ at sacrifice $\left(1345.2 \pm 685.2\right.$ and $1144.0 \pm 213.1$ vs $375.7 \pm 114.9 \mathrm{~mm}^{3}$, respectively, $p<0.01$ ) (Fig. $3 \mathrm{~b}, \mathrm{c}$ ). Immunohistochemistry analysis showed a robust decrease in the number of Ki67+ cells in EFtargeted tumor sections ( $90 \%$ and $88 \%$ vs $12 \%, p<0.001$ ) and an increase in the number of apoptotic cells ( $9 \%$ and $10 \%$ vs $45 \%, p$ $<0.001$ ) (Fig. 3d, e). Of note, $40 \%$ of xenografted mice treated with AdCas9_EF survived beyond day 80, whereas all PBS- or AdCas9_NT-treated mice succumbed to the disease by day 40 (Fig. 3f). Hematoxylin and eosin (H\&E) staining of tumors revealed a significantly lower number of viable tumor cells and more extensive necrotic regions in EF-targeted tumors (Supplementary Fig. 5a). Immunohistochemistry for Cas 9 confirmed adenoviral delivery into the tumors (Fig. 3d). Immunohistochemostry analysis of the tumors from AdCas9_EF-treated mice showed the colocalization of adenovirus expression (Cas9 immunostaining) with high and low expression of caspase- 3 and Ki67, respectively (Fig. 3g). Because we used the Hds:Athymic Nude-Foxn1nu mouse strain, which lacks $\mathrm{T}$ cells and shows a partial defect in B cell development (with a weakened innate immune system), we tested for the presence of tumor-infiltrating CD45 + leukocyte cells. Results showed that the necrotic region was surrounded by tumor-infiltrating murine CD45 + leukocytes (Fig. 3g). Targeted deep-sequencing analysis of the tumors at sacrifice revealed that $E F$-targeted xenografted tumors analyzed 6 weeks after AdV injection were composed of non-edited (85\%) or partially-edited (one locus; $12 \%$ of EWSR1 or 3\% of FLI1) cancer cells (Supplementary Table 2e, f). No cells harboring an EF deletion were present in the tumors suggesting that CRISPRmediated abrogation of $E F$ is sufficient to inhibit survival of Ewing sarcoma cells in vivo. Furthermore, targeted deepsequencing of PCR amplicons covering the 50 most probable predicted off-target sites associated with sgE3 and sgF8 ruled out mutations in EF-targeted xenografted tumors analyzed 6 weeks after AdV injection (Supplementary Table 3).

FO deletion controls tumor growth in PDX models. To further investigate the utility of the approach, we used three patientderived xenograft (PDX) models of Ewing sarcoma. The models were established by subcutaneous implantation of Ewing sarcoma PDXs into immunodeficient mice, which were allowed to develop for 12 days (tumors $\sim 150 \mathrm{~mm}^{3}$ in size) (Fig. 4a). FISH analysis showed the presence of the $t(11 ; 22)$ translocation (Supplementary Fig. 5b). We next examined the capacity of FO targeted deletions to reduce PDX growth by measuring tumor size. As described earlier, EWSR1-FLI1 targeted therapy was administered by adenoviral delivery using $2.5 \times 10^{9}$ pfu of the AdCas9_EF in $100 \mu \mathrm{L}$ of PBS (or a PBS control) at day 12, 14, 16 and 19 (Fig. 4a). 
a

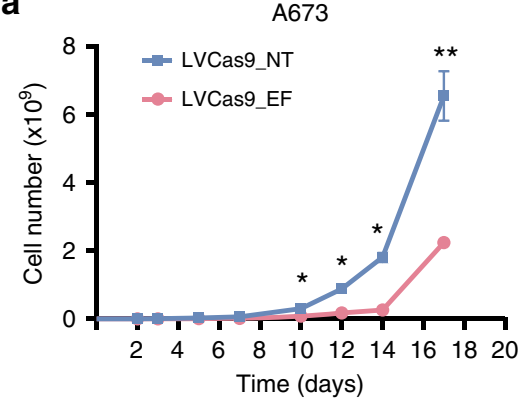

d

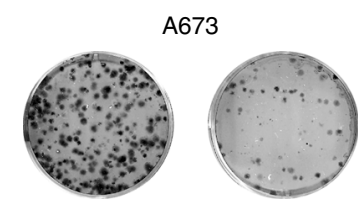

LVCas9_NT LVCas9_EF
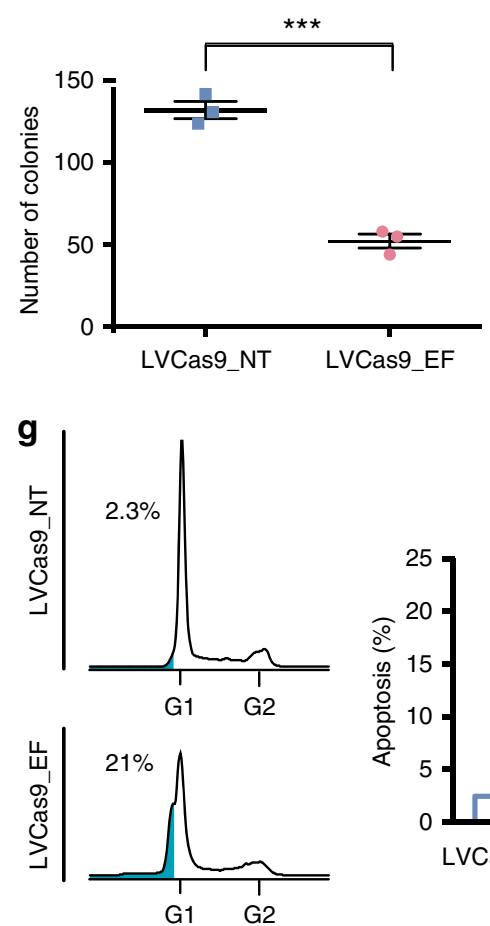

b

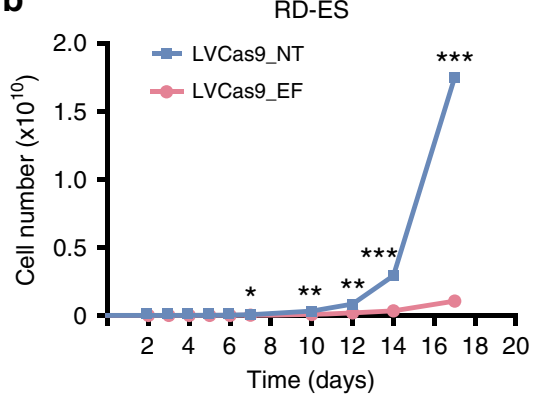

e
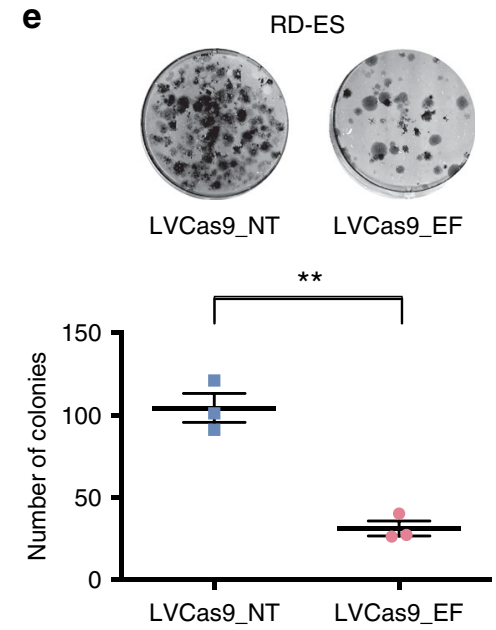

h
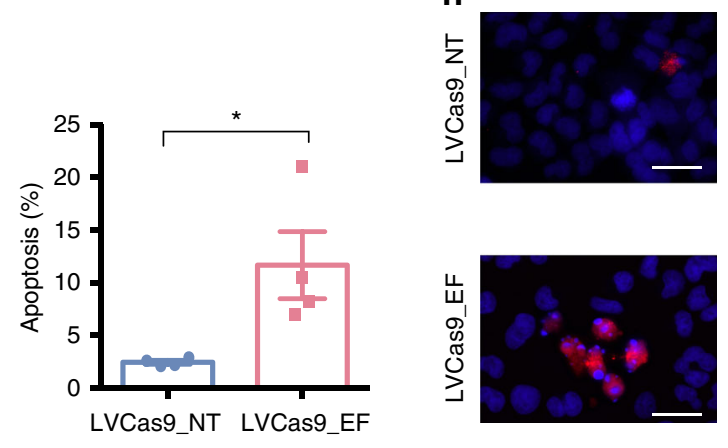

c

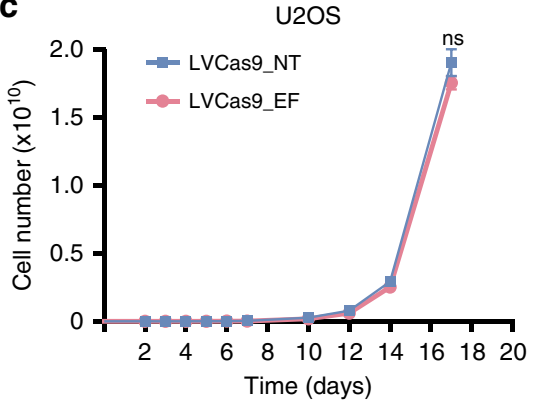

f
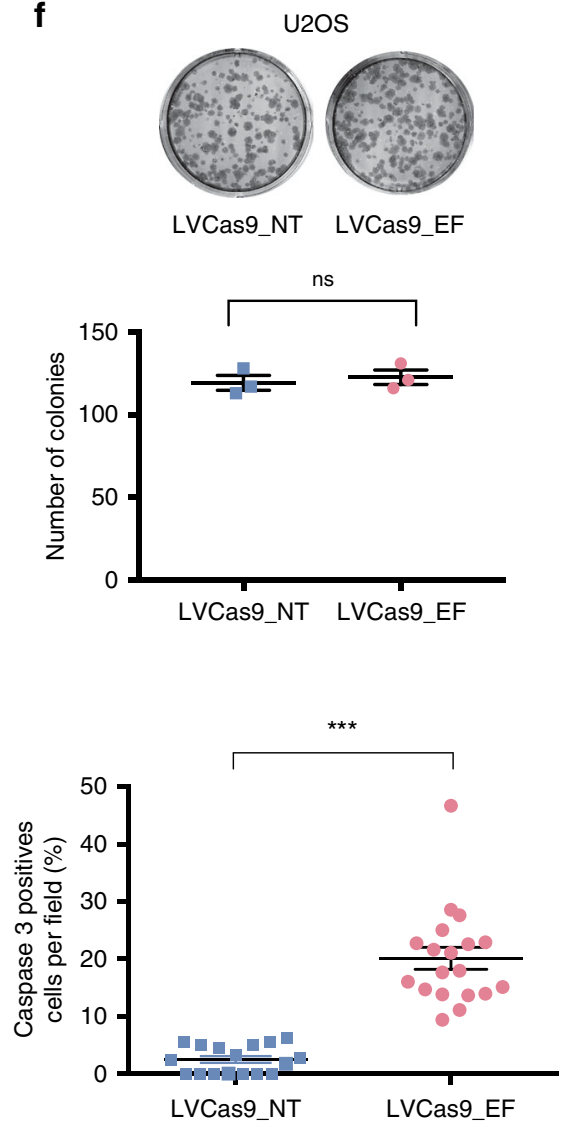

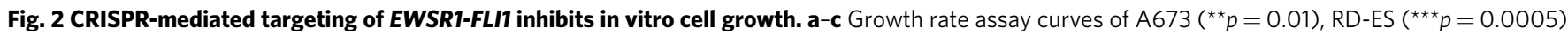
and U2OS edited (LVCas9_EF) and control (LVCas9_NT) cells. ( $n=6$ independent experiments). d-f Representative crystal violet staining of A673 $\left({ }^{\star \star *} p=0.0003\right), \mathrm{RD}-\mathrm{ES}\left({ }^{\star \star} p=0.0017\right)$ and U2OS experimental and control cells. Bottom panels show the statistical analysis of the number of colonies. ( $n=3$ independent experiments). $\mathbf{g}$ Left panel shows DNA profile analysis by propidium iodide staining and flow cytometry. FlowJo v10.0.7 flow cytometry analysis of DNA fragmentation by subG1 population (cells with fragmented DNA) quantification. The percentage of cellular apoptosis was calculated using the percentage of the subG1 peak. Right panel shows graphical representation of subG1 analysis. $\left(n=4\right.$ independent experiments) ${ }^{*} p=0.027$. h Representative images of caspase-3-immunostained A673 cells. Scale bars, $50 \mu \mathrm{m}$. Right panel shows the percentage of caspase-3-positive cells per field analyzed. ( $n=520$ (LVCas9_NT) and $n=613$ (LVCas9-EF) cells examined over three independent experiments), ${ }^{\star \star \star} p=1.07 \mathrm{e}-9$. The error bars indicate the s.e.m. for the averages across the multiple experiments; $p$-values are represented (ns non-significant; ${ }^{\star} p \leq 0.05 ;{ }^{\star \star} p \leq 0.01 ;{ }^{\star \star \star} p \leq 0.001$ ). Statistical are two-tailed unpaired $t$-test.

Results showed that tumors were $>74 \%$ smaller in AdCas9_EFtreated mice at sacrifice than in PBS-treated mice $(334.87 \pm 28.01$ vs $1321.70 \pm 249.77 \mathrm{~mm}^{3}, p<0.001$ ) (Fig. $4 \mathrm{~b}$, c). Immunohistochemistry analysis of tumor sections showed significantly fewer Ki67+ cells in $E F$-targeted tumors $(18 \%$ vs $73 \%, p<0.001)$ and an increased number of apoptotic cells $(57 \%$ vs $1.5 \%, p<0.001)$ (Fig. 4d, e). H\&E staining also revealed a significantly lower number of viable tumor cells and more extensive necrotic regions in EF-targeted tumors (Fig. 4d). Immunohistochemistry for Cas9 confirmed adenoviral delivery into the tumors (Fig. 4d). Of note, $100 \%$ of PDX mice treated with AdCas9_EF survived beyond day $52 \%$ and $50 \%$ beyond day 70 , whereas all PBS control mice succumbed to the disease by day 40 (Fig. $4 \mathrm{f}$ ). Taken together, these results show that in vivo adenoviral delivery of $E F$-targeting CRISPR components controls sarcoma growth.

Combined deletion and chemotherapy enhances tumor regression. We next investigated the possible synergy between 
a

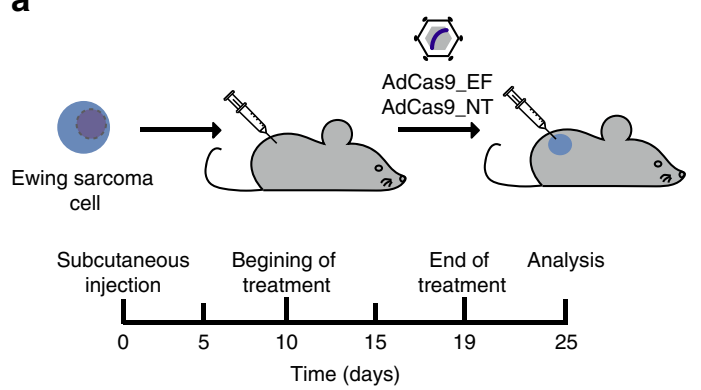

d

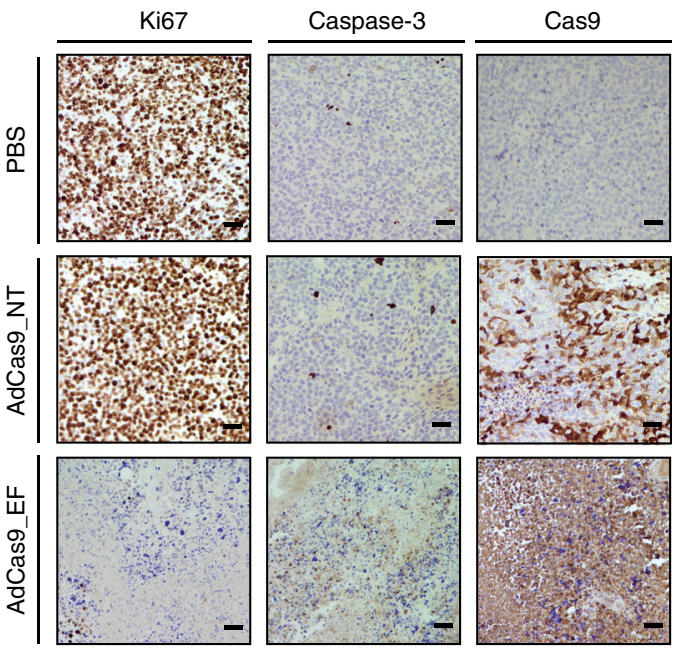

b

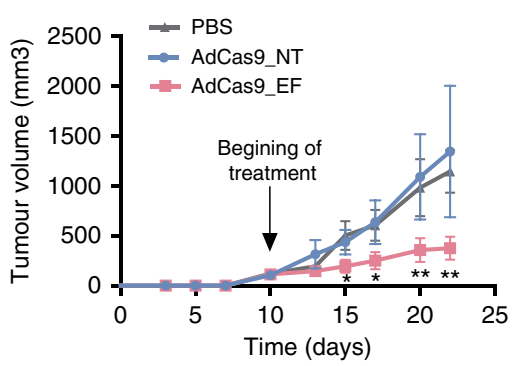

C PBS AdCas9 NT
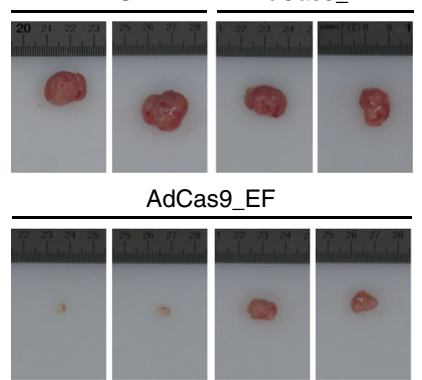

e

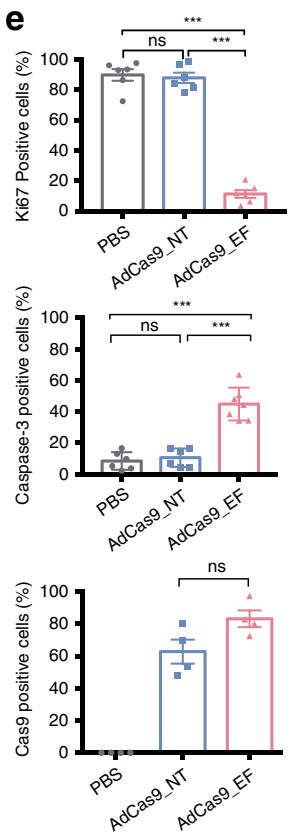

f
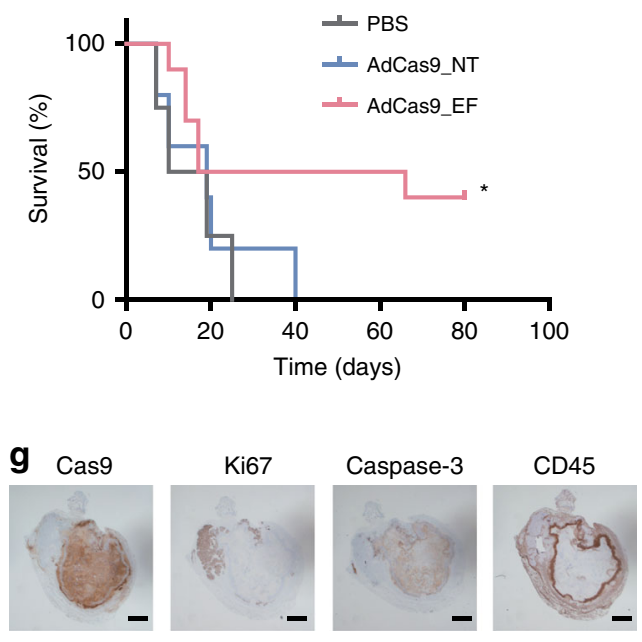

Fig. 3 Deletion of EWSR1-FLI1 inhibits tumor growth in xenografted models. a Diagram showing the approach for the in vivo xenograft treatment. A673 cells were subcutaneously injected into immunodeficient mice and tumors were allowed to develop ( $150 \mathrm{~mm}^{3}$ in size). Tumors were then injected with AdCas9_EF edition vector, AdCas9_NT control vector or PBS at days 10, 13, 16, and 19. Mice were sacrificed when tumor volume reached 1500 mm ${ }^{3}$. b Tumor growth over 23 days of analysis. (PBS $n=6$; AdCas9_NT $n=6$; AdCas9_EF $n=15$ animals), ${ }^{\star \star} p=0.004$. c Images of representative tumors. d Representative immunostaining for Ki-67, caspase-3 and Cas 9 in A673 experimental and control tumors $(n=3$ independent samples). Scale bars, $50 \mu$ m. e Statistical analysis of the percentage of Ki-67- (PBS vs AdCas9_EF ${ }^{\star \star \star} p=1 e-8$, AdCas9_NT vs AdCas9_EF ${ }^{\star \star \star} p=6 e-9$ ), caspase-3- (PBS vs AdCas9_EF ${ }^{\star \star \star} p=1 \mathrm{e}-5$, AdCas9_NT vs AdCas9_EF ${ }^{\star \star \star} p=5 \mathrm{e}-5$ ) and Cas9-positive cells. $\mathbf{f}$ Kaplan-Meier survival curve comparing mice treated with experimental and control AdV or PBS. (PBS $n=4$; AdCas9_NT $n=5$; AdCas9_EF $n=10$ animals), ${ }^{\star} p=0.046$. g Representative immunostaining for Cas9, Ki-67, caspase-3, and CD45 in an AdCas9_EF-treated xenografted tumor. The error bars indicate the s.e.m. for the averages across the multiple experiments; $p$-values are represented (ns non-significant; ${ }^{*} p \leq 0.05 ;{ }^{* \star} p \leq 0.01 ;{ }^{* \star} p \leq 0.001$ ). A two-tailed unpaired $t$-test was used for statistical analysis of $\mathbf{b}, \mathbf{d}$, and log-rank test for $\mathbf{f}$.

our genomic editing approach to efficiently eliminate cancer cells and a standard-of-care chemotherapeutic option for Ewing sarcoma with doxorubicin. We examined the additive effects of combined doxorubicin and $E F$ deletion both in vitro and in vivo. Corresponding FO deletion and treatment of A673 cells with 0.05 $\mu \mathrm{g} / \mathrm{ml}$ doxorubicin resulted in a greater reduction of cell viability (50\%) than single treatments (39\% or $42 \%$ with AdCas9_EF or doxorubicin treatment alone, respectively) (Fig. 5a). For in vivo analysis, we utilized the xenograft models generated as described earlier, divided into four treatment groups. Mice were treated with $1.5 \mathrm{mg} / \mathrm{kg}$ doxorubicin (intraperitoneal injection, weekly, for two weeks) or with $2.5 \times 10^{9}$ pfu of the AdCas9_EF or AdCas9_NT virus in PBS (intratumour injection, at day 9, 12, 15, and 18) (Fig. 5b). Three groups received monotherapy of AdCas9_NT, doxorubicin or AdCas9_EF, while the remaining group was treated with combined therapy (doxorubicin + AdCas9_EF), and animals were monitored for 80 days. Results showed that mice treated with combined therapy showed a greater reduction in tumor size at sacrifice $(92.12 \%, 115.28 \pm$ $\left.28.35 \mathrm{~mm}^{3}\right)$ than mice treated with monotherapy $(83.7 \%, 237.4 \pm$ $47.39 \mathrm{~mm}^{3}$ AdCas9_EF or $70.4 \%, 433.05 \pm 56.91 \mathrm{~mm}^{3}$ doxorubicin) as compared with control mice $\left(1463.82 \pm 278.17 \mathrm{~mm}^{3}\right)$ (Fig. 5c). These results indicate that combined therapy is more effective than monotherapy. Of note, $66 \%$ of xenografted mice treated with combined therapy survived beyond day 80 compared with 33\% of AdCas9_EF-treated mice. By contrast, all the mice that received doxorubicin monotherapy succumbed to the disease by day 64 (Fig. 5d).

Validation as a method for elimination of FO-addicted cells. To validate the editing strategy as a universal strategy for elimination of FO-driven cancer cells, we applied the same approach in a cellular model harboring $B C R-A B L 1(B A)$, a classical tyrosine kinase FO hallmark of chronic myeloid leukemia (CML). 
a

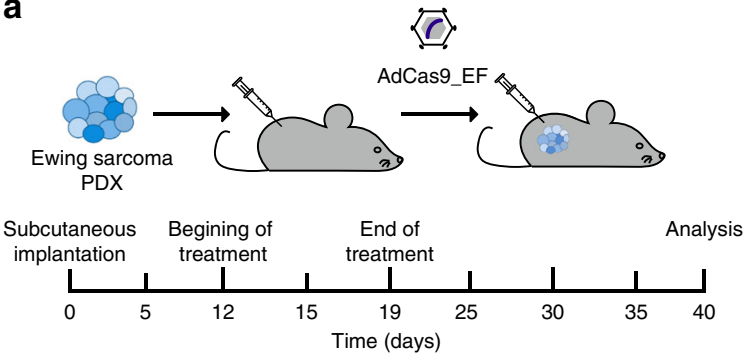

d
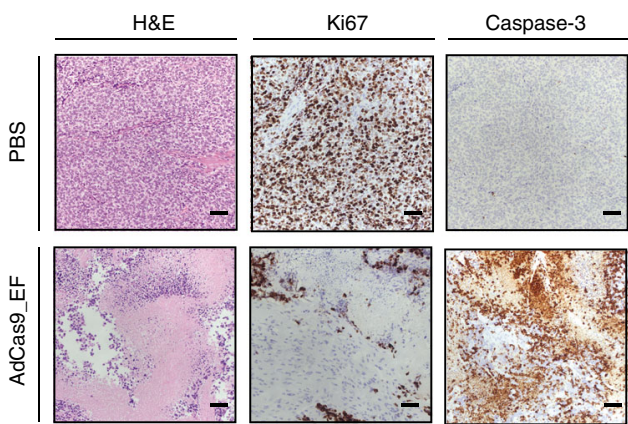

b

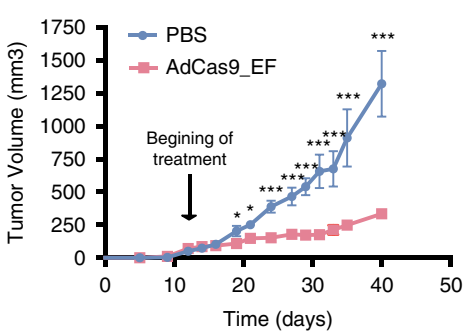

c
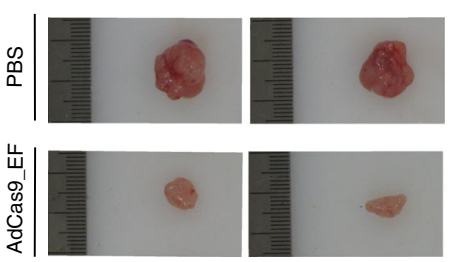

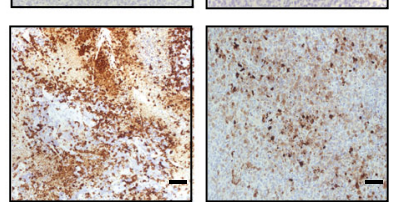

e
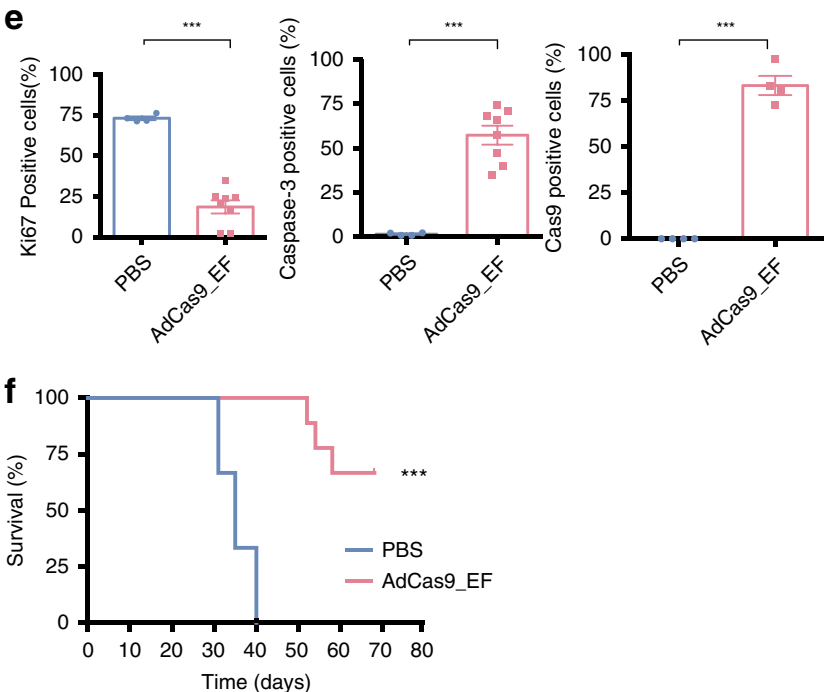

Fig. 4 Deletion of EWSR1-FLI1 inhibits tumor growth in PDX models. a Diagram showing the approach for the AdV-based in vivo treatment. PDXs were implanted into immunosuppressed mice and tumors were allowed to develop ( $150 \mathrm{~mm}^{3}$ in size). Tumors were then injected with AdCas9_EF edition vector or PBS control vector at days 12,14,16, and 19. Mice were sacrificed when tumor volume reached $1500 \mathrm{~mm}^{3}$. b Tumor growth over the 40 days following subcutaneous PDX implantation. (PBS $n=3$; AdCas9_sgEF $n=9$ animals) $\left.{ }^{\star \star \star} p=3 e-5\right)$. c Representative tumors of control and experimental mice sacrificed 40 days after PDX implantation. $\mathbf{d}$ Representative images of histological H\&E staining, Ki-67, caspase-3, and Cas 9 immunostaining of A673

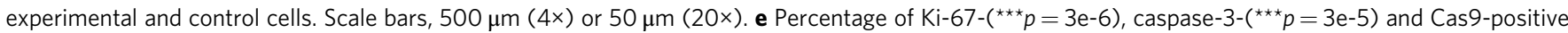
cells per field analyzed ( $\left.{ }^{\star \star \star} p=4 \mathrm{e}-6\right)$. ( $n=3$ independent samples). f Kaplan-Meier survival curve comparing mice treated with experimental and control AdV. (PBS $n=3$; AdCas9_sgEF $n=9$ animals), ${ }^{\star \star \star} p=0.0002$. Plot shows medians and ranges; error bars indicate the s.e.m. for the averages across the multiple experiments; $p$-values are represented $\left({ }^{\star} p \leq 0.05,{ }^{* *} p \leq 0.01,{ }^{\star \star \star} p \leq 0.001\right)$. A two-tailed unpaired $t$-test was used for statistical analysis of b, e, and log-rank test for $\mathbf{f}$

Four sgRNAs targeting $B C R$ intron 8 and $A B L$ intron 1 regions were designed to induce a $133.9 \mathrm{~kb}$ deletion of the $B A \mathrm{FO}$ (Supplementary Table 1), resulting in an extensive deletion of the $B C R$ transactivation domain and a frameshift alteration of the entire ABL1 DNA-binding domain (Fig. 6a and Supplementary Fig. 3b). Four sgRNA combinations (sgB8.1-sgA1.1, sgB8.1sgA1.2, $\mathrm{sgB8} .2 \mathrm{sgA1} .1$, and $\mathrm{sgB8} .2$ - $\mathrm{sgA1}$.2) were cloned into the pLV-U6 ${ }^{\mathrm{B}} \mathrm{H} 1^{\mathrm{A}}-\mathrm{C} 9 \mathrm{G}$ vector as before, and were electroporated into the $B A$-expressing CML cell line K562 (Fig. 6b). Electroporation was chosen as the delivery method because of the low transduction efficiency of lentiviral vectors in K562 cells ${ }^{39}$. All four sgRNA combinations efficiently abrogated the expression of $B A$ as confirmed by RT-PCR and Sanger sequencing (Fig. 6c). For any given sgRNA combination, electroporated K562 cells showed a significant decrease $(\sim 85 \%)$ in clonogenic capacity in vitro (Fig. 6d) concomitant with an increase in apoptosis (3\% vs $15 \%$, $p<0.001)$ relative to equivalent cells electroporated with a nontargeting vector (Supplementary Fig. 6a). The use of a control plasmid (LVCas9_dC3/4) targeting two unrelated genomic loci in two different chromosomes ruled out any effects on cell viability in K562-treated cells due to the induction of two DSBs (Supplementary Table 1 and Supplementary Fig. 6b, c). Because cleavage with sgB8.1-sgA1.1 resulted in the highest reduction in colony formation and the highest percentage of apoptosis rate this combination was chosen for further study. For subsequent targeting of $B A$, sgB8.1 and sgA1.1 were cloned into an all-in-one expression plasmid ${ }^{34}$ (pLVCas9_BA). Targeted deep-sequencing of sgB8.1 or sgA1.1-targeted K562 cells revealed $85 \%$ and $82 \%$ indels in $B C R$ and $A B L 1$ on-target sites, respectively (Supplementary Table 2). Similarly, targeted deep-sequencing of PCR amplicons covering the most probable predicted off-target sites associated with sgB8.1 and sgA1.1 ruled out mutations in $B A$ targeted K562 cells after pLVCas9_BA electroporation (Supplementary Table 3). qRT-PCR analysis of FO expression at day 2 post-electroporation (p.e.) of pLVCas9_BA edited K562 cells used to estimate rates of deletion revealed a significant 5.0-fold decrease in FO expression (Fig. 6e). We also analyzed the presence of inversions in K562 cells as before. PCR analysis revealed the presence of inversion products at 2 days p.e.; however, inversion carrier cells were lost during cell culture and no inversion products were detectable at day 7 p.e. (Supplementary Fig. 6d). In silico analysis showed that an inversion of the FO targeted region will produce a truncated protein similar to the one induced by DNA deletion (Supplementary Fig. 3b). We used cord blood-derived human hematopoietic progenitor hCD34+ cells as a control cell of origin for CML. In vitro analysis of targeted 
a

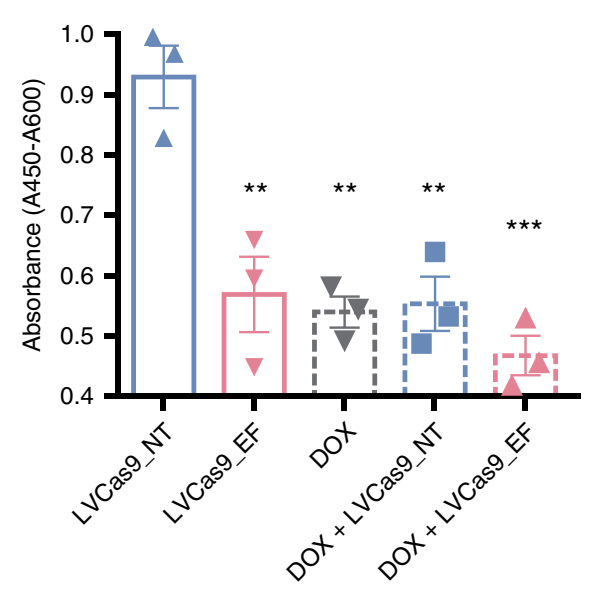

C

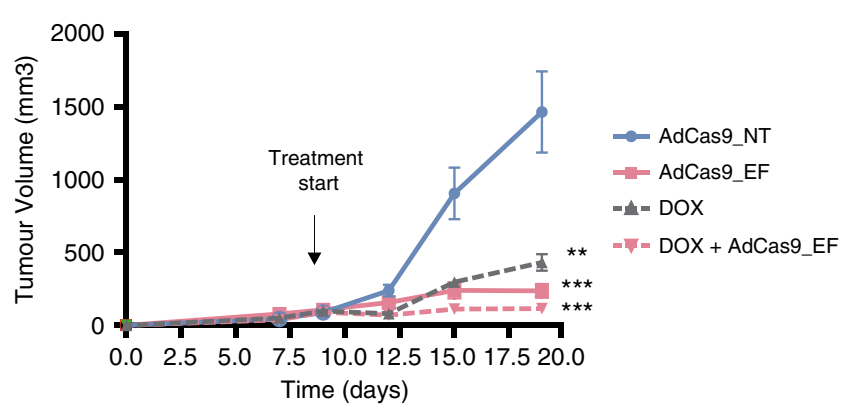

b
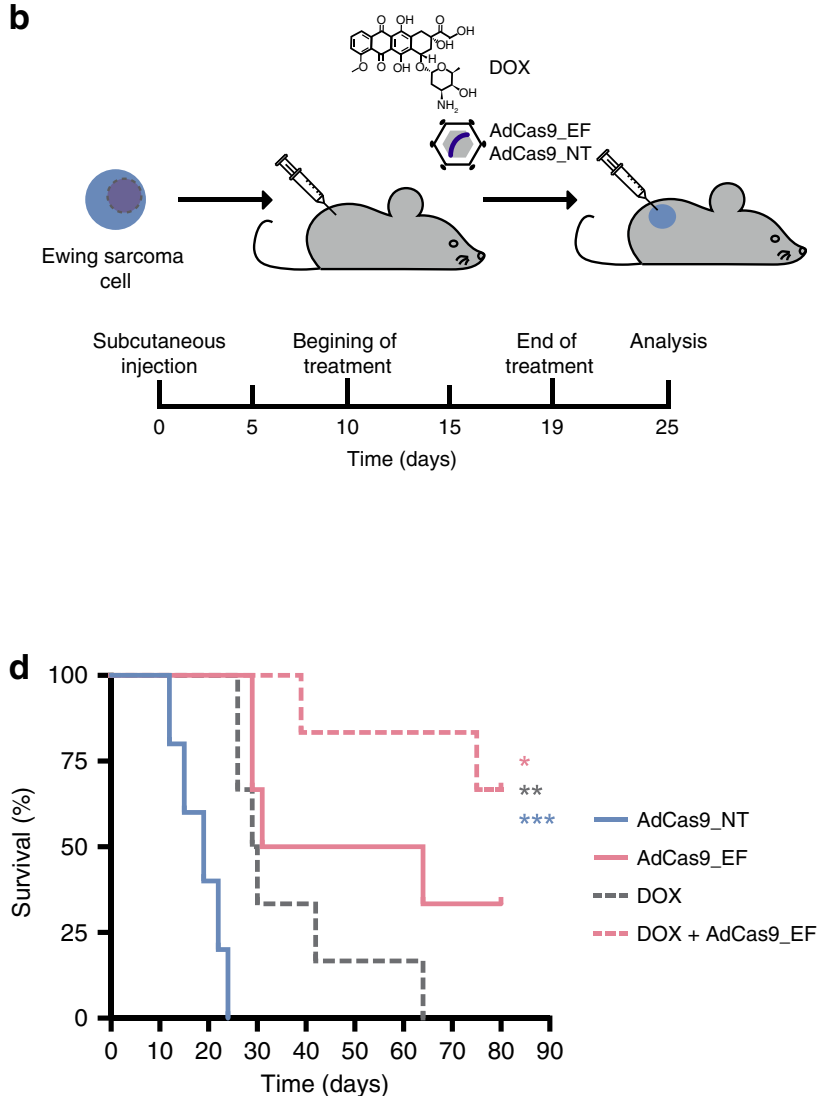

Fig. 5 Combined approach of EWSR1-FLI1 deletion with doxorubicin inhibits tumor growth in xenograft models. a WST-1 cell proliferation analysis of A673 cell treated with CRISPR-deletion or doxorubicin as monotherapy, or as combined therapy and controls $(n=3$ independent experiments) (LVCas9_NT vs LVCas9_EF ${ }^{* *} p=0.01$, LVCas9_NT vs Dox ** $p=0.002$, LVCas9_NT vs Dox + LVCas9_NT ** $p=0.005$, LVCas9_NT vs Dox + LVCas9_EF $\left.{ }^{* \star *} p=0.0001\right)$. b Diagram showing the approach for the in vivo xenograft treatment. A673 cells were subcutaneously injected into immunodeficient mice and tumors were allowed to develop $\left(150 \mathrm{~mm}^{3}\right.$ in size). Tumors were then injected with AdCas9_EF edition vector, doxorubicin (DOX), AdCas9_NT control vector or their combination at days 9, 12, 15, and 18. Mice were sacrificed when tumor volume reached 1500 mm ${ }^{3}$. c Tumor growth over the 19 days following subcutaneous cells implantation. (AdCas9_NT $n=5$; AdCas9_sgEF $n=6$; DOX $n=6$, DOX + AdCas9_EF $n=6$ animals) (AdCas9_NT vs DOX ${ }^{\star \star} p=0.003$, AdCas9_NT vs AdCas9_EF ${ }^{\star \star *} p=9 \mathrm{e}-4$, AdCas9_NT vs DOX + AdCas9_EF ${ }^{\star \star \star} p=5 \mathrm{e}-4$ ). d Kaplan-Meier survival curve comparing mice treated with CRISPR-deletion or DOX as monotherapy, or as combined therapy and controls. (AdCas9_NT $n=5$; AdCas9_sgEF $n=$ 6; DOX $n=6$, DOX + AdCas9_EF $n=6$ animals) (DOX + AdCas9_EF vs AdCas9_EF * $p=0.035, \mathrm{DOX}+\mathrm{AdCas9 \_ EF} \mathrm{vs} \mathrm{DOX} \mathrm{**} p=0.003, \mathrm{DOX}+$ AdCas9_EF vs AdCas9_NT ${ }^{* \star *} p=0.0007$ ). Plot shows medians and ranges; error bars indicate the s.e.m. for the averages across the multiple experiments; $p$-values are represented $\left({ }^{\star} p \leq 0.05,{ }^{\star \star} p \leq 0.01,{ }^{\star \star \star} p \leq 0.001\right)$. A two-tailed unpaired $t$-test was used for statistical analysis of $\mathbf{a}, \mathbf{c}$, and Logrank test for $\mathbf{d}$.

$\mathrm{CD} 34+$ cells revealed no difference in proliferation in longterm culture, suggesting no production of collateral cancerdriven genomic alterations (Supplementary Fig. 6e). No BCR$A B L 1$ translocation products were detected in cells by PCR or FISH assays conducted at 15- and 30-days p.e. (Supplementary Fig. 6f, g, Supplementary Table 1). We then evaluated whether AdV delivery of the $B A$-targeting CRISPR machinery (sgB8.1sgA1.1) also controls the growth of $B A$-driven tumors in vivo. Accordingly, K562 cells were subcutaneously injected into immunodeficient mice and tumors were allowed to develop for 15 days $\left(\sim 150 \mathrm{~mm}^{3}\right.$ in size) (Fig. $\left.6 \mathrm{f}\right)$. Tumors were then injected with $2.5 \times 10^{9}$ pfu of the AdCas9_BA editing vector or the AdCas9_NT control vector at day 15, 18, and 21. Adenoviral delivery of AdCas9_BA profoundly inhibited tumor growth, resulting in an $88 \%$ decrease in tumor size at sacrifice $(281 \pm 63$ vs $1152 \pm 158 \mathrm{~mm}^{3}(p<0.001)$ compared with control tumors (Fig. $6 \mathrm{~g}, \mathrm{~h}$ ). Strikingly, $50 \%$ and $22 \%$ of xenografted mice treated with AdCas9_BA were alive after 38 and 64 days, respectively, whereas all AdCas9_NT-treated mice succumbed to the disease by day 34 (Fig. 6i). As observed in the Ewing sarcoma model, the efficiency of the AdCas9_BA editing vector was also associated with a robust decrease in the number of Ki67+ cells ( $82 \%$ vs $17 \%, p<0.001)$ and an increase in apoptotic cells $(2.5 \%$ vs $10 \%, p<0.05)$ (Supplementary Fig. 6 h, i). $\mathrm{H} \& \mathrm{E}$ staining also revealed a significantly lower number of viable tumor cells and more extensive necrotic regions in $B A$ targeted tumors (Supplementary Fig. 6h). A combinatorial approach using $B A$ deletion with a standard-of-care therapeutic option in CML (imatinib, $0.105 \mu \mathrm{g} / \mathrm{ml}$ ) resulted in a significant reduction in $\mathrm{K} 562$ cell viability compared with any of the treatments in monotherapy (Fig. 6j). Targeted deep-sequencing analysis of the tumors at sacrifice revealed that $B A$-targeted xenografted tumors analyzed 6 weeks after AdV injection were composed of non-edited (94\%) or partially-edited (one locus; $3 \%$ of $B C R$ or $A B L 1$ ) cancer cells (Supplementary Table $2 \mathrm{~g}, \mathrm{~h}$ ). Taken together, these results confirm the efficacy of CRISPRmediated targeting of sarcoma $(E F)$ - and leukemia $(B A)$-associated FO for cancer cell-directed elimination. 
a

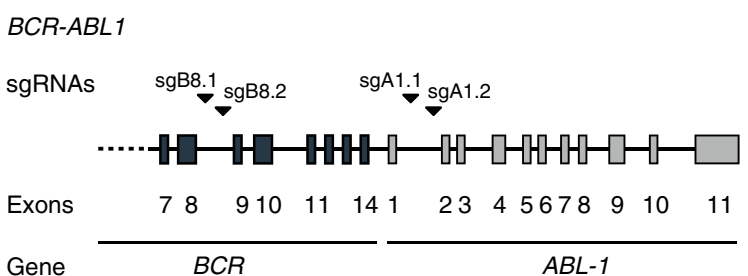

C
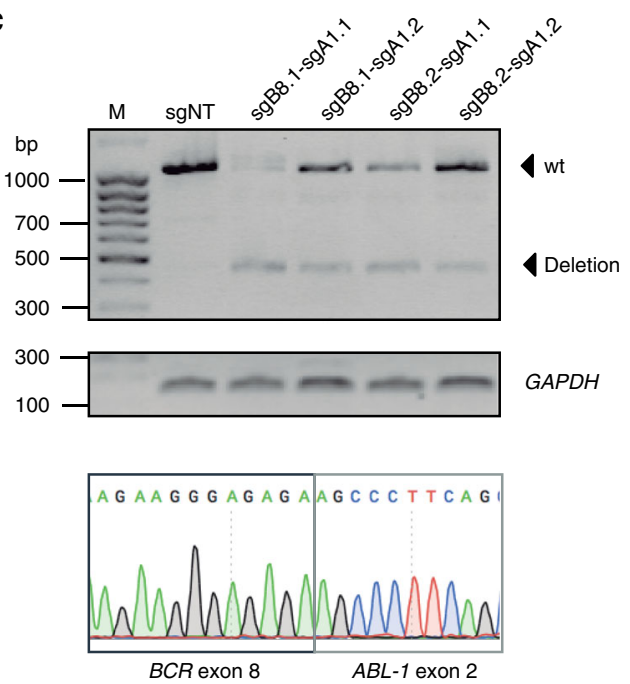

f

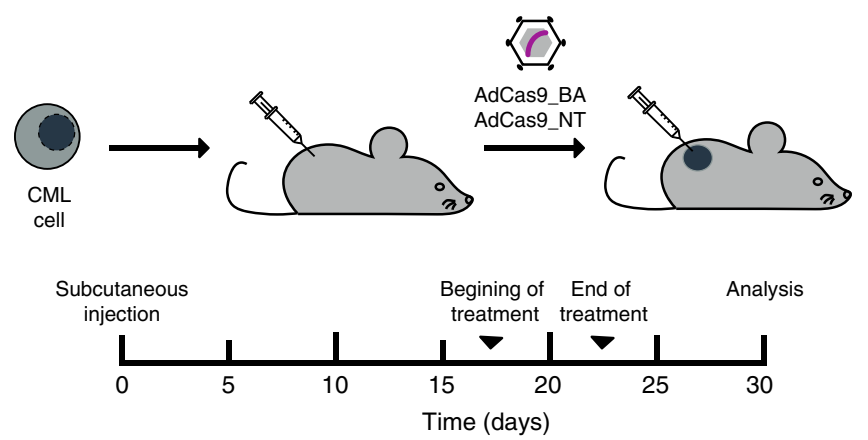

h

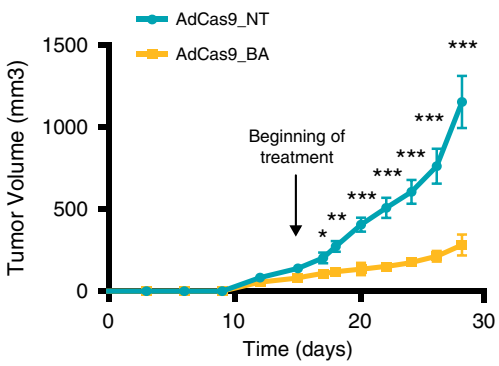

i

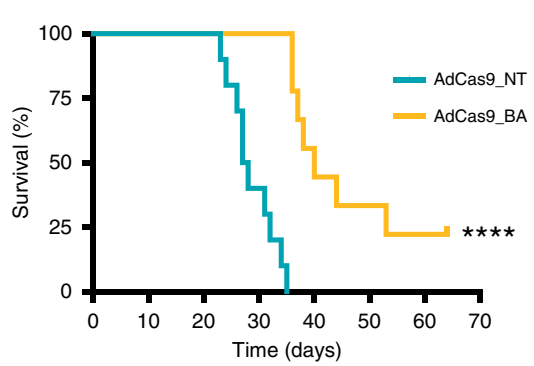

b

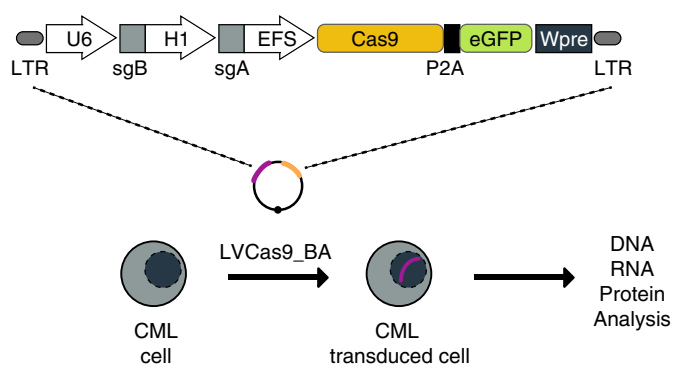

e
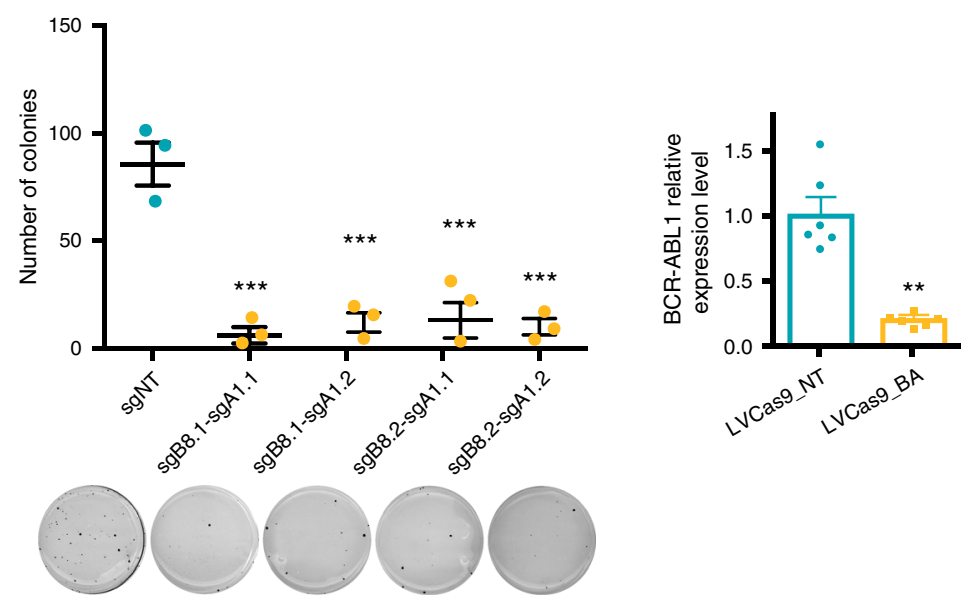

g
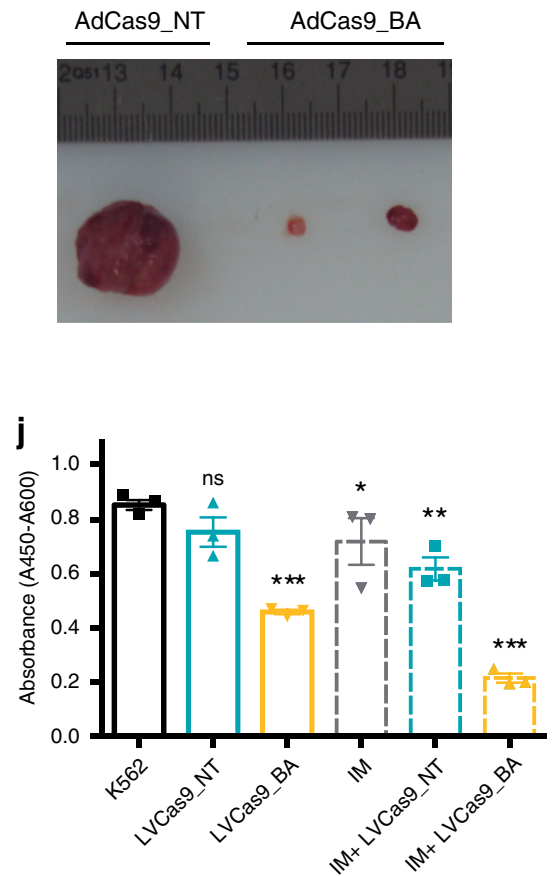

\section{Discussion}

FOs are ideal therapeutic targets for the development of new directed cancer treatments, owing to their cancer-driving roles, their restriction to cancer cells and the reliance of tumors on them. Unfortunately, FOs are challenging to target directly with candidate drugs, and although some successful anti-cancer drugs have been developed based on the ability to target $\mathrm{FOs}^{40,41}$, the development of new therapies are still needed. The ability to precisely manipulate cancer cell genomes to correct or eliminate cancer-causing aberrations by highly-efficient CRISPR/Cas9 genome editing opens new possibilities to develop FO-targeted options to eliminate cancer cells. In the present study, we describe a simple and efficient genome editing strategy specifically targeting FOs in cancer cells. Our CRISPR/Cas9-based approach 
Fig. 6 Strategy validation in CML-initiating BCR-ABL model. a Schematic representation of the $B C R$ - $A B L 1$ locus showing the sgRNAs targeting introns 8 of $B C R$ and 1 of $A B L 1$. b Schematic illustration of the vector and the approach for in vitro treatment. c Agarose gel electrophoresis of BCR-ABL RT-PCR products obtained from experimental and control K562 cells electroporated with four combinations of sgRNAs. RT-PCR analysis was done using RNA extracted from cells at day 2 post-nucleofection. Arrows depict the sizes of wild-type (1125 bp) and deleted (458 bp) RT-PCR products. GAPDH was used as an internal control of the RT-PCR reaction. Bottom panel shows a representative chromatogram of Sanger sequencing analysis of the RT-PCR products. d, Representative images and graphical representation of colony formation of $\mathrm{K} 562$ experimental and control cells (stained with nitrotetrazolium blue). ( $n=3$ independent experiments). sgNT vs sgB8.1-agA1.1 ${ }^{\star \star \star} p=3 e-10$, sgNT vs sgB8.1-agA1.2 ${ }^{\star \star *} p=5 \mathrm{e}-4$, sgNT vs sgB8.2-sgA1.1 ${ }^{\star \star \star} p=3 \mathrm{e}-4$, sgNT vs sgB8.2-agA1.2 ${ }^{\star \star} p=1$ e-4. e $B C R 1-A B L$ expression level analysis. Relative expression level of $B C R-A B L 1$ in control (LVCas9_NT) and treated (LVCas9_EF) K562 cells measured by qRT-PCR and normalized to GUSB ( $n=6$ independent experiments, $\left.{ }^{\star \star} p=0.0020\right)$. $\mathbf{f}$ Diagram of xenograft production, adenoviral treatment and tumor growth over the 30 days following subcutaneous cell injection and four in vivo adenoviral treatments. (AdCas9_NT $n=10$;

AdCAas9_sgBA $n=10$ ). $\mathbf{g}$ Representative tumors of control and experimental mice. $\mathbf{h}$ Tumor growth curve over the 28 days of study. (AdCas9_NT $n=10$; AdCas9_sgBA $n=10,{ }^{\star \star \star} p=3 e-4$ ). i Kaplan-Meier survival curve comparing mice treated with experimental and control adenoviral vectors (AdCas9_NT $n=10$; AdCas9_sgBA $n=10$ animals, $\left.{ }^{\star \star \star \star} p<0.0001\right)$. $\mathbf{j}$ WST-1 cell proliferation analysis of K562 cell treated with CRISPR-deletion or imatinib (IM) as monotherapy, or as combined therapy and controls ( $n=3$ independent experiments), K562 vs LVCas9_BA ${ }^{\star \star \star} p=3.9 \mathrm{e}-5, \mathrm{~K} 562$ vs IM ${ }^{\star} p=0.043, \mathrm{~K} 562$ vs IM + LVCas9_NT ${ }^{* *} p=0.006$, K562 vs IM + LVCas9_BA ${ }^{* \star *} p=4 \mathrm{e}-6$. Plot shows medians and ranges; error bars indicate the s.e.m. for the averages across the multiple experiments; $p$-values are represented $\left({ }^{\star} p \leq 0.05,{ }^{\star \star} p \leq 0.01,{ }^{\star \star \star} p \leq 0.001\right)$. A two-tailed unpaired $t$-test was used for statistical analysis of $\mathbf{d}$, $\mathbf{e}, \mathbf{h}$ and $\mathbf{j}$, and Log-rank test for $\mathbf{i}$.

induces two targeted intronic DSBs in both genes involved in a FO that, importantly, produces a cancer cell-specific genomic deletion that is dependent on the presence of the FO, and has no effect on wild-type gene expression in non-cancer cells. By targeting two established FOs, involving either a transcription factor $(E F)$ or a tyrosine kinase $(B A)$, we present a proof-of-concept demonstration supporting the efficacy of genome edition to eliminate cancer cells addicted to FOs. Exploiting an adenoviral in vivo delivery of CRISPR components, we further demonstrate that CRISPR/Cas9-mediated deletion in advance PDX cancer models results in efficient induction of cancer cell death and reduces tumor burden and mortality. Finally, we show that targeted CRISPR-based FO deletion combined with chemotherapy agents has an additive/synergistic effect on cell viability, tumor growth and overall survival when used in xenograft models. In the long term, this approach may provide an attractive strategy to eliminate cancer cells and we believe our findings might have applicability to other FOs beyond $E F$ and $B A$. Due to the complex genomic scenario in cancer cells, including co-existence of other oncogenes, tumor suppressor genes, DNA damage response and DNA repair pathways alterations, a case-by-case FO study could shed light to the applicability of the strategy to eliminate different types of cancer cells.

A recent study has described an approach for targeting FOs using CRISPR/Cas9-based editing and the HDR DNA repair mechanism. The strategy involves directly targeting the patientspecific breakpoint to introduce a suicide gene into the genome of cancer cells ${ }^{25}$. Notably, this elegant approach is based on poorly efficient and breakpoint-specific design. Our system offers unique advantages over breakpoint-targeted approaches. First, it exploits the efficient NHEJ pathway active in all cell types, making the design simple and highly efficient ${ }^{42}$, as shown here with high efficiency targeting FOs for in vivo elimination of cancer cells with significant decreases in tumor volume and increases in survival rates. Second, the strategy targets two introns flanking all breakpoint regions described in patients, making it a universal approach for all cancer cells harboring a given FO irrespective of the FO isoform or the patient-specific breakpoint. For those promiscuous FOs (such as EWSR1) that have been described rearranged with many different fusion partners, a combination of the original $5^{\prime}$ sgRNA with a set of pre-designed $3^{\prime}$ sgRNAs will cover all the possible FO family ${ }^{31}$. Third, our approach does not affect exonic sequences or the expression of wild-type alleles involved in the rearrangement.

CRISPR/Cas9-mediated genome editing could be a potential platform for cancer treatment; however, (i) targeting efficiencies, (ii) off-target effects, and (iii) in vivo delivery remain technical limitations. As described in the present study, combinatorial approaches using gene editing with other therapeutic options (e.g., chemotherapy) further increases the efficacy of this approach, allowing better responses with lower chemotherapy doses for the elimination of cancer cells. In contrast to chemotherapy agents, which can be damaging to normal tissues, we show that the CRISPR approach is completely specific for the elimination of cancer cells ${ }^{43,44}$. Potential off-target events at unintended genomic sites remain important concerns that must be investigated rigorously to ensure short- and long-term safety of any gene-editing approach. We have used in vitro and in vivo assays to study the potential effect of collateral cancer-driver genomic alterations. Even though our results did not show proliferation differences, these experiments are limited, and further analysis will be needed to rule out the appearance of cancerdriven off-target modifications. Although the CRISPR-based toolbox supports diverse operations ranging from DNA and RNA editing to gene expression modulation, delivery remains a bottleneck for therapy development ${ }^{45}$. AdV vectors are a commonly utilized delivery vector in mouse models; they are ideal for delivery of the entire CRISPR/Cas9 system in one vector, with high transfection efficacy and they limit potential off-target effects because their genome does not integrate into the host genome ${ }^{46-48}$. Although the initially encountered high immunogenicity inhibited their widespread use to treat genetic disorders, the overall picture changed when it was recognized that adenoviral infection in a tumor can activate a robust immunogenic response, creating new opportunities in cancer therapy 49,50 . The number of clinical trials using oncolytic AdVs is increasing, and at present more than 32 trials are underway in different phases (https://clinicaltrials.gov) ${ }^{51}$. As in our study, the main delivery pathway used in these clinical trials is local administration (intratumoral injection), which shows no toxicity at specific doses and, in addition to the therapeutic effect, triggers an antitumor immune response with therapeutic effects even on not-injected distal tumors ${ }^{52}$. Our Hds:Athymic Nude-Foxn1 animal model characterized by partial defects in B cell development shows tumor-infiltrating CD45 + mouse leukocyte cells in the tumor. Nevertheless, additional studies on immunocompetent syngeneic mouse models could yield information about the role of AdVs as a delivery tool in cancer therapy. In the present study, we used adenoviral-based in vivo delivery of the CRISPR/Cas9 components as a proof-of-concept; however, we envision that other delivery platforms such as AAVs and non-viral (polymeric and liposome-based) vectors could be exploited to deliver the system 
to tumor cells. Further development of safer and robust delivery systems and the refinement of genome editing technologies will ultimately ensure optimal targeting efficiencies and safety required for the potential clinical application of this cancer celldirected therapy.

Overall, our data demonstrate that NHEJ CRISPR-mediated FO deletion is a selective and efficient strategy for cancer cell elimination, providing a valuable tool for basic research and could represents a promising strategy with therapeutic potential.

\section{Methods \\ sgRNA design and generation of lentiviral constructs. sgRNAs were designed using the online Benchling CRISPR gRNA Design tool (http://www.benchling. com). The sgRNAs chosen were based on a high specificity rank and a low potential off-target score ${ }^{53}$. The parental Streptococcus pyogenes Cas9 expression lentiviral single and double sgRNAs expression vectors, pLV-sgRNA-Cas9 and pLV-U6\#1-H1\#2-C9G, respectively, have been described ${ }^{33,34}$. sgRNAs were cloned using synthetic gBlock fragments (IDT) that were digested with PacI and XhoI (NEB) to facilitate cloning into the lentiviral backbone. When two different guides were used, the first was cloned under the control of the U6 promoter and the second was cloned under the control of the $\mathrm{H} 1$ promoter. The sequences for sgRNAs, gBlocks, and primers used are listed in Supplementary Table 1. All transformations were performed in Stbl3 bacteria (Thermo Fisher Scientific).}

Cell culture. HEK293T/17 (CRL-11268), A673 (CRL-1598), umbilical cord bloodderived hMSC (PCS-500-010), U2OS (HTB-96), and K562 (CCL-243) cells were purchased from the American Type Culture Collection (ATCC). RD-ES cells were a gift from Dr. Javier Alonso (Instituto Salud Carlos III, Madrid). HEK293T/17, U2OS, and A673 cells were maintained in Dulbecco's modified Eagle's medium (DMEM, Lonza) and K562 and RD-ES cells were maintained in Roswell Park Memorial Institute (RPMI) medium (Gibco); all were supplemented with $1 \%$ Glutamax (Life Technologies), $10 \mathrm{mg} / \mathrm{ml}$ antibiotics (penicillin and streptomycin, $\mathrm{P} / \mathrm{S}$, Gibco) and 10\% fetal bovine serum (FBS, Life Technologies). hMSCs were cultured in MesenPRO RS media (Thermo Fisher Scientific) supplemented with 1\% Glutamax (Life Technologies) and $10 \mathrm{mg} / \mathrm{ml}$ antibiotics (P/S, Gibco). hCD34+ were obtained freshly from the umbilical cord blood of healthy donors after informed consent was obtained and after approval by the Cord Blood Bank Transfusion Center of the Community of Madrid. Mononuclear cells were purified by gradient centrifugation (Histopaque, Sigma-Aldrich) and $\mathrm{hCD} 34^{+}$cells were purified by separation using magnetic beads (Miltenyi Biotec) ${ }^{54} \cdot \mathrm{hCD} 34+$ cells were cultured in StemSpan SFEM II (StemCell Technologies) containing 100 U/ $\mathrm{mL} / \mathrm{S}$ and a cytokine cocktail composed of $100 \mathrm{ng} / \mathrm{mL}$ of human stem cell factor (hSCF) human FMS-like tyrosine kinase 3 (hFlt3L) and human thrombopoietin (hTPO) and $10 \mathrm{ng} / \mathrm{mL}$ of human interleukin 3 (hIL3) (all from Preprotech). Cells were cultured at $37^{\circ} \mathrm{C}$ at $5 \% \mathrm{CO}_{2}, 5 \% \mathrm{O}_{2}$ atmosphere in a humidified incubator. All cell lines were periodically tested for mycoplasma contamination.

Lentivirus generation, titration, transduction. Recombinant lentiviruses were produced by transient plasmid transfection of HEK293T/17 cells ${ }^{55}$. Briefly, cells were seeded at $1.1 \times 10^{7}$ cells/dish in $15-\mathrm{cm}$ dishes the day before transfection, and were transfected by the calcium-phosphate method using 14.6 and $7.9 \mu \mathrm{g}$ of second-generation packaging plasmids (psPAX2 and pMD.2G, Addgene \#12260 and \#12259, respectively) and $22.5 \mu \mathrm{g}$ of the appropriate transfer plasmid depending on the experiment. The medium was collected after $48 \mathrm{~h}$, cleared by low-speed centrifugation, and filtered through $0.45 \mu \mathrm{m}$ PVDF filters (Millipore) Viral stocks were concentrated by ultracentrifugation in a Beckman LE ultracentrifuge using a SW28 rotor at $20,000 \times g$ for $2 \mathrm{~h}$ at $4{ }^{\circ} \mathrm{C}$. Pellets containing lentivirus were air dried and resuspended overnight at $4^{\circ} \mathrm{C}$ in $300 \mu \mathrm{L}$ of sterile $\mathrm{NaCl} 0.9 \%$ solution (B.Braun). Viral titers were calculated by FACS analysis on transduced HEK293T cells when vectors expressed fluorescent proteins (transduction units $/ \mathrm{mL}$ ) and particles quantified by qPCR in supernatants (particles $/ \mathrm{mL}$ ), and were between $10^{7}$ and $10^{8} \mathrm{TU} / \mathrm{mL}$. Viral aliquots were stored at $-80^{\circ} \mathrm{C}$. Target cells were re-plated $24 \mathrm{~h}$ before transduction and transduced using a multiplicity of infection between 2 and 5 . Cells were incubated at $37^{\circ} \mathrm{C}$ for $6 \mathrm{~h}$ and then viral supernatant were replaced with fresh media.

In vitro colony forming assays. For $\mathrm{A} 673$, RD-ES and U2OS cells, colony forming assays were performed $24 \mathrm{~h}$ after transduction with the corresponding lentiviral vectors in six-well plates $(500 \text { cells/well })^{56}$. Cells were allowed to grow for 14-21 days, after which cells were fixed using methanol solution and stained with a $0.5 \%$ crystal violet solution (Sigma-Aldrich) and counted. For K562 cells, methylcellulose (StemCell Technologies) assays were performed by seeding 500 cells into 35-mm tissue culture dishes (ThermoFisher Scientific) $24 \mathrm{~h}$ after electroporation. Colonies were stained two weeks later with Nitrotetrazolium Blue chloride solution (NBT, Sigma-Aldrich) for $1 \mathrm{~h}$ at $37^{\circ} \mathrm{C}$ and counted. All assays were performed in triplicate and repeated three times.
Apoptotic SubG1 cell detection. Cell cycle distribution was analyzed by measuring the DNA content using flow cytometry. Exponentially growing transduced cells were pelleted by centrifugation, washed with PBS (Sigma-Aldrich), resuspended in ice-cold $70 \%$ ethanol (Sigma-Aldrich) in PBS for fixation, and were maintained at $4{ }^{\circ} \mathrm{C}$ for $24 \mathrm{~h}$. Prior to analysis, fixed cells were washed with phosphate-citrate buffer (Sigma-Aldrich) and incubated with propidium iodide staining solution (Sigma-Aldrich) containing $100 \mu \mathrm{g} / \mathrm{mL}$ of RNase (Qiagen) for 30 min. Cellular DNA content was measured using a BD FACSCanto ${ }^{\mathrm{TM}}$ platform (BD Biosciences) and analyzed by using FlowJo $^{\circledR}$ software (version 10.0.6, FlowJo).

PCR, RT-PCR, and qRT-PCR analysis. Genomic DNA and total RNA were extracted using standard procedures with the DNeasy Blood and Tissue Kit or the RNeasy Purification Kit, respectively (Qiagen). DNA and RNA were quantified by spectrophotometry. cDNA was generated from $1 \mu \mathrm{g}$ of total RNA using the HighCapacity cDNA Reverse Transcription Kit (ThermoFisher Scientific). PCR and RTPCR amplification were performed using Q5 High-Fidelity DNA Polymerase (NEB). qRT-PCR was performed in 384-well plates with 2x SYBR Green Master Mix (Applied Biosystems) using a QuantStudio 6 Detection System (Applied Biosystems). Expression levels were normalized to the housekeeping genes ALBUMIN for genomic DNA PCR and GAPDH for RT-PCR or GUSB for qRTPCR. The primers used are listed in Supplementary Table 1.

Western blotting. Proteins were extracted using standard procedures in the presence of protease inhibitor ${ }^{57}$. Protein lysates were fractionated by SDS-PAGE and transferred to PVDF membranes (Millipore) using TransFi (ThermoFisher Scientific). Membranes were probed for FLIl with a mouse monoclonal antibody (1/400; BD Pharmigen). GAPDH was used as a loading control (1/200; Abcam). Secondary antibodies were HRP-conjugated with a goat anti-mouse IgG $(1 / 1000$; Abcam) and blots were developed with the ECL reagent (GE Healthcare) and exposed to film (Kodak).

Immunoassays. To detect apoptosis, transduced A673 cells were seeded onto glass coverslips coated with poly-L-lysine (Cultek). After $72 \mathrm{~h}$, cells were washed twice with PBS (Sigma-Aldrich), fixed in 4\% paraformaldehyde (PFA; Electron Microscope Sci) for $12 \mathrm{~min}$ at room temperature (RT), permeabilized with $0.3 \%$ Triton X-100 (Sigma-Aldrich) in PBS and blocked with 3\% normal goat serum (NGS; Sigma-Aldrich) in PBS for $1 \mathrm{~h}$ at RT. Thereafter, samples were incubated overnigh at $4{ }^{\circ} \mathrm{C}$ with an anti-caspase-3 antibody (1/200; BD) diluted in PBS supplemented with $1 \%$ NGS, and then with an Alexa Fluor-594-conjugated secondary antibody (1/500; ThermoFisher Scientific) for $1 \mathrm{~h}$ at RT. Finally, samples were counterstained with DAPI (Vector Labs), air dried and mounted in Vectashield mounting medium (Vector Labs). Images were acquired on a Leica DM5500B microscope (Leica Microsystems) with two lasers with excitation at $594 \mathrm{~nm}$ (red channel, caspase-3 detection) and $405 \mathrm{~nm}$ (blue channel, nuclear DAPI staining). Data were collected sequentially at a resolution of $1024 \times 1024$ pixels and are representative of every experiment analyzed using Cytovision v7.4 software (Leica Biosystems).

Tumor tissues were fixed with 4\% PFA (Electron Microscopy Sci) in PBS overnight at $4{ }^{\circ} \mathrm{C}$ for immunohistochemistry assays ${ }^{58}$. Tissue sections were deparaffinized, rehydrated, blocked with $3 \%$ hydrogen peroxide (Merck) and subjected to heat-induced antigen retrieval. The following antibodies were used at the indicated dilutions: mouse anti-Ki-67 (DAKO; 1:1.000); rabbit anti-cleaved caspase-3 (1:800); rabbit anti-GFP (1:1000), mouse anti-Cas9 (1:1000) and Rabbit anti-CD45 (D3F8Q) (1:200) (all from Cell Signaling Technology). Immunostained tissue images were examined with an Olympus AX70 microscope and processed with ImageJ to estimate the labeling index (percentage of positively stained nuclear area).

Karyotyping. Cell cultures were arrested in metaphase by incubation with KaryoMAX Colcemid (Gibco; $0.1 \mathrm{mg} / \mathrm{mL}$ ). Cells were treated with a hypotonic solution $\left(0.075 \mathrm{M} \mathrm{KCl}\right.$ in ddH2O; Merck) for $30 \mathrm{~min}$ at $37^{\circ} \mathrm{C}$ and were then fixed with Carnoy's solution (methanol and acetic acid 3:1; Sigma-Aldrich). For GTG banding, slides were trypsinized (ThermoFisher Scientific) and stained with Giemsa (ThermoFisher Scientific). Metaphase spreads were analyzed on a Zeiss Axioplan microscope with Ikaros v5.20 karyotyping platform (Metasystems $\mathrm{GmbH})$. Between 40 and 100 metaphases were analyzed.

Comparative genomic hybridization arrays. Comparative genome hybridization analysis was performed on a dual color oligonucleotide-based array (SurePrint G3 Human CGH Array $8 \times 60 \mathrm{~K}$; Agilent) with a $41 \mathrm{~kb}$ overall median probe spacing (Agilent). Sample and reference DNA (Agilent) samples were labeled with Cy5 and Cy3 dyes (Agilent), respectively. Hybridization was performed according to the manufacturer's protocols. Arrays were scanned using the G2565BA DNA Microarray Scanner (Agilent Technologies) and data were extracted using Feature Extraction Software v10.7 (Agilent Technologies). The Aberration Detection Method 2 (ADM-2) algorithm in Cytogenomics v5.0 (Agilent Technologies) was used to identify contiguous genomic regions corresponding to chromosomal aberrations or copy number variations, which were scored based on the average quality weighted log ratio of the sample and reference channels. 
Fluorescence in situ hybridization. The EWSR1 break-apart (Kreatech), EWSR1FLI1 dual-fusion (Cytocell) and BCR-ABL1 dual-fusion FISH probe (Kreatech) FISH probes were used to detect $t(11 ; 22)$ and $t(9 ; 22)$ chromosomal translocations. Tissue sections $(5 \mathrm{~mm})$ were deparaffinized in xylene and rehydrated in ethanol (Sigma-Aldrich). Tissue sections were pre-treated in 2-[N-morpholino] ethanesulphonic acid (DAKO), followed by pepsin digestion (DAKO). After dehydration, the samples were denatured in the presence of the appropriate probe at $66^{\circ} \mathrm{C}$ for $10 \mathrm{~min}$ and left overnight for hybridization at $37^{\circ} \mathrm{C}$ in a hybridizer machine (DAKO). The slides were then washed with $20 x S S C / T w e e n 20$ buffer at $63^{\circ} \mathrm{C}$ and mounted on fluorescence mounting medium (with DAPI). FISH signals were manually scored by counting the number of nuclei with split signals across the tissue. FISH images were captured using a CCD camera (Photometrics SenSys camera) connected to a PC running the Zytovision image analysis system (Applied Imaging Ltd., UK).

Targeted deep sequencing. Potential off-target sites were predicted using the CRISPR Design web server (http://crispr.mit.edu) ${ }^{53}$, and Cas-OFFinder (http:// www.rgenome.net/cas-offinder/ $/)^{59}$. Customized PCR primers for the on-target and top-50 potential off-target sites to amplify the sgRNA target region were ordered from IDT with rhAmpSeq index sequences (Supplementary Table 1). Each locus was individually amplified using genomic DNA extracted from the relevant cells. First PCR was performed rhAmpSeq Library Kit (IDT) under the following conditions: $95^{\circ} \mathrm{C}$ for $10 \mathrm{~min}, 10$ cycles of $95^{\circ} \mathrm{C}$ for $15 \mathrm{~s}$ and $61{ }^{\circ} \mathrm{C}$ for 4 min and $99.5^{\circ} \mathrm{C}$ for $15 \mathrm{~min}$. PCR products of all potential off-target loci were subsequently equally pooled and purified with Agencourt AMPure XP beads (Beckman). The PCR products were then subjected to a second PCR with Illumina indexed adaptor primers under the following PCR conditions: $95^{\circ} \mathrm{C}$ for $3 \mathrm{~min}, 18$ cycles of $95^{\circ} \mathrm{C}$ for $15 \mathrm{~s}, 60^{\circ} \mathrm{C}$ for $30 \mathrm{~s}$ and $72{ }^{\circ} \mathrm{C}$ for $30 \mathrm{~s}$, and $72^{\circ} \mathrm{C}$ for $1 \mathrm{~min}$. Second PCR products were purified with Agencourt AMPure XP beads (Beckman) at a PCR product:bead ratio of 1:1. The purified libraries were quantified and sequenced using a 300-bp paired-end cycle on the Illumina MiSeq sequencing platform. Results were analyzed with CRISPR RGEN Tools Cas-Analyser software ${ }^{60}$.

Animal experiments. All experiments with mice conformed to Animal Welfare guidelines and were performed in accordance to protocols approved by the Ethics Committee of the Instituto de Salud Carlos III. Tumors were generated in 8-weekold athymic nude (Hds:Athymic Nude-Foxn1 nu) female mice (Charles River) by subcutaneous implantation of $7.5 \times 10^{5}$ cells in BD Matrigel (volume ratio 1:1). PDX models were established from patient biopsies (HSJD-ES-006, HSJD-ES-013, HSJD-ES-018) at Sant Joan de Déu Hospital (Barcelona, Spain). Cells were implanted into the flanks of 8 -week-old athymic nude mice. Tumor growth was measured blinded to the experimental conditions at the indicated time intervals. Tumor volume was estimated using a caliper and calculated as volume $\left(\mathrm{mm}^{3}\right)=$ (length $[\mathrm{mm}]) \times(\text { width }[\mathrm{mm}])^{2} \times 0.52$. When tumors reached $\sim 150 \mathrm{~mm}^{3}$, mice were separated blinded to the experimental groups and treated accordingly. Adenoviral inoculation consisted of a total of $2.5 \times 10^{9} \mathrm{pfu}(100 \mu \mathrm{L}$ total volume) administered in $3 / 4$ doses every 3 days. Two doses, one per week, of $1.5 \mathrm{mg} / \mathrm{kg}$ of doxorubicin ([DOX], S1208, Selleckchem) were administered in xenografted mice. In some experiments, when tumors reached $1500 \mathrm{~mm}^{3}$ in size, mice were euthanized and tumors were surgically excised and processed for histopathology.

Adenovirus production. Viral vectors and viruses were constructed and produced by the Universitat Autonoma de Barcelona (UAB) viral vector production unit. Briefly, homologous recombination in $E$. coli between shuttle plasmids and fulllength adenovirus backbones (E1-deleted) were used for the generation of recombinant adenoviral vectors genomes. The adenovirus genomes were transfected to generate a recombinant adenoviral vector in permissive human cells, and purified by two sequential $\mathrm{CsCl}$ gradient centrifugations and subjected to chromatography to eliminate the $\mathrm{CsCl}$ and exchange buffers. Viral titers were calculated and were between $10^{11}$ and $10^{12}$ particles/ml. Finally, the viral stocks were characterized through the quantification of viral particle content and infectivity. Viral aliquots were stored at $-80^{\circ} \mathrm{C}$ before injection.

Electroporation. The Neon Transfection System (Thermo Fisher Scientific) was used for cell electroporation ${ }^{34}$. Confluent K562 cells were passed into fresh media $24 \mathrm{~h}$ prior to electroporation at a low density; the next day cells were resuspended in $\mathrm{T}$ solution and electroporated using the established conditions by the manufacturer: $10 \mu \mathrm{L}$ tips were used to electroporate $4 \times 10^{5}$ cells with four 10 -ms pulses at $1350 \mathrm{~V}$. After electroporation, cells were seeded in 24-well plates containing prewarmed medium.

WST-1 measurement. For the chemotherapy study, $0.05 \mu \mathrm{g} / \mathrm{ml}$ of doxorubicin ([DOX], S1208, Selleckchem), and $0.105 \mu \mathrm{g} / \mathrm{ml}$ of imatinib ([IM], S2475, Selleckchem) were used for in vitro assays of Ewing sarcoma and CML, respectively. WST-1 measurements were performed according to the standard protocol of the manufacturer. Briefly, A673 or K562 cells (10,000/well) were cultured in 96-well plates and overnight. The next day, doxorubicin or imatinib were added to A673 and K562 cells, respectively, and plates were incubated for $24 \mathrm{~h}$. Then $10 \mu \mathrm{l}$ of
WST- 1 was added and plates were incubated for a further $3 \mathrm{~h}$ at $37^{\circ} \mathrm{C}$ in the incubator. The absorbance was monitored at $450 \mathrm{~nm}$.

Statistical analysis. Statistical analysis was performed using GraphPad Prism software package (version 7.0, GraphPad Software). Data from three or more independent experiments were analyzed by two-tailed Student's unpaired $t$-test. Statistical details of the experiments can be found in the corresponding figure legends. To flag levels of significance, asterisks were used as follow $\left(^{*}\right) p$-value less or equal than $0.05,(* *) p$-value $\leq 0.01$, and $\left({ }^{* *}\right) p$-value $\leq 0.001$ and (ns) was used when non-significant differences were observed.

Reporting summary. Further information on research design is available in the Nature Research Reporting Summary linked to this article.

\section{Data availability}

The NGS data have been deposited in the BioProject database under the accession code PRJNA659633. All other data supporting the findings of this study are available within the article and its Supplementary Information files and from the corresponding author upon reasonable request.

Received: 23 December 2019; Accepted: 16 September 2020; Published online: 08 October 2020

\section{References}

1. Hsu, P. D., Lander, E. S. \& Zhang, F. Development and applications of CRISPR-Cas9 for genome engineering. Cell 157, 1262-1278 (2014).

2. $\mathrm{Lu}, \mathrm{H}$. et al. Engineering and functional characterization of fusion genes identifies novel oncogenic drivers of cancer. Cancer Res. 77, 3502-3512 (2017).

3. Rabbitts, T. H. Chromosomal translocations in human cancer. Nature $\mathbf{3 7 2}$, 143-149 (1994).

4. Rodriguez-García, A. et al. Selective destruction of tumor cells through specific inhibition of products resulting from chromosomal translocations. Curr. Cancer Drug Targets 1, 109-119 (2001).

5. Thomas, M., Greil, J. \& Heidenreich, O. Targeting leukemic fusion proteins with small interfering RNAs: recent advances and therapeutic potentials. Acta Pharmacol. Sin. 27, 273-281 (2006).

6. Yoshihara, K. et al. The landscape and therapeutic relevance of cancerassociated transcript fusions. Oncogene 34, 4845-4854 (2015).

7. Andersson, A. K. et al. The landscape of somatic mutations in infant MLLrearranged acute lymphoblastic leukemias. Nat. Genet. 47, 330-337 (2015).

8. Gao, Q. et al. Driver fusions and their implications in the development and treatment of human cancers. Cell Rep. 23, 227-238.e3 (2018).

9. Tomlins, S. A. et al. Recurrent fusion of TMPRSS2 and ETS transcription factor genes in prostate cancer. Science 310, 644-648 (2005).

10. Chou, A. et al. NTRK gene rearrangements are highly enriched in MLH1/ PMS2 deficient, BRAF wild-type colorectal carcinomas-a study of 4569 cases. Mod. Pathol. 33, 924-932 (2020).

11. Banerji, S. et al. Sequence analysis of mutations and translocations across breast cancer subtypes. Nature 486, 405-409 (2012).

12. Ahmed, A. A. \& Abedalthagafi, M. Cancer diagnostics: the journey from histomorphology to molecular profiling. Oncotarget 7, 58696-58708 (2016).

13. Mitelman, F., Johansson, B. \& Mertens, F. The impact of translocations and gene fusions on cancer causation. Nat. Rev. Cancer 7, 233-245 (2007).

14. Mertens, F., Johansson, B., Fioretos, T. \& Mitelman, F. The emerging complexity of gene fusions in cancer. Nat. Rev. Cancer 15, 371-381 (2015).

15. Rabbitts, T. H. \& Stocks, M. R. Chromosomal translocation products engender new intracellular therapeutic technologies. Nat. Med. 9, 383-386 (2003).

16. Cierpicki, T. \& Grembecka, J. Targeting protein-protein interactions in hematologic malignancies: still a challenge or a great opportunity for future therapies? Immunol. Rev. 263, 279-301 (2015).

17. Tanaka, T. \& Rabbitts, T. H. Intrabodies based on intracellular capture frameworks that bind the RAS protein with high affinity and impair oncogenic transformation. EMBO J. 22, 1025-1035 (2003).

18. Jiang, F. et al. Progress and challenges in developing aptamer-functionalized targeted drug delivery systems. Int. J. Mol. Sci. 16, 23784-23822 (2015).

19. Kim, J. E., Yoon, S., Mok, H., Jung, W. \& Kim, D.-E. Site-specific cleavage of mutant ABL mRNA by DNAzyme is facilitated by peptide nucleic acid binding to RNA substrate. FEBS Lett. 586, 3865-3869 (2012).

20. Kuwabara, T. et al. A novel allosterically trans-activated ribozyme, the maxizyme, with exceptional specificity in vitro and in vivo. Mol. Cell 2, 617-627 (1998).

21. Borkhardt, A. Blocking oncogenes in malignant cells by RNA interference-new hope for a highly specific cancer treatment? Cancer Cell 2, 167-168 (2002). 
22. Martinez-Lage, M., Puig-Serra, P., Menendez, P., Torres-Ruiz, R. \& Rodriguez-Perales, S. CRISPR/Cas9 for cancer therapy: hopes and challenges. Biomedicines 6, 105 (2018).

23. Cong, L. et al. Multiplex genome engineering using CRISPR/Cas systems. Science 339, 819-823 (2013).

24. Mali, P. et al. RNA-guided human genome engineering via Cas9. Science 339, 823-826 (2013).

25. Chen, Z.-H. et al. Targeting genomic rearrangements in tumor cells through Cas9-mediated insertion of a suicide gene. Nat. Biotechnol. 35, 543-550 (2017).

26. Liu, M. et al. Methodologies for Improving HDR Efficiency. Front Genet. 9, 691 (2018).

27. Bailly, R. A. et al. DNA-binding and transcriptional activation properties of the EWS-FLI-1 fusion protein resulting from the $t(11 ; 22)$ translocation in Ewing sarcoma. Mol. Cell. Biol. 14, 3230-3241 (1994).

28. May, W. A. et al. The Ewing's sarcoma EWS/FLI-1 fusion gene encodes a more potent transcriptional activator and is a more powerful transforming gene than FLI-1. Mol. Cell. Biol. 13, 7393-7398 (1993).

29. May, W. A. et al. Ewing sarcoma 11;22 translocation produces a chimeric transcription factor that requires the DNA-binding domain encoded by FLI1 for transformation. Proc. Natl Acad. Sci. USA 90, 5752-5756 (1993).

30. Grohar, P. J. et al. Functional genomic screening reveals splicing of the EWSFLI1 fusion transcript as a vulnerability in ewing sarcoma. Cell Rep. 14, 598-610 (2016).

31. Sankar, S. \& Lessnick, S. L. Promiscuous partnerships in Ewing's sarcoma. Cancer Genet. 204, 351-365 (2011).

32. Cantile, M. et al. Molecular detection and targeting of EWSR1 fusion transcripts in soft tissue tumors. Med. Oncol. 30, 412-415 (2013).

33. Torres, R. et al. Engineering human tumour-associated chromosomal translocations with the RNA-guided CRISPR-Cas9 system. Nat. Commun. 5, 3964 (2014).

34. Torres-Ruiz, R. et al. Efficient recreation of $t(11 ; 22)$ EWSR1-FLI1(+) in human stem cells using CRISPR/Cas9. Stem Cell Rep. 8, 1408-1420 (2017).

35. Levetzow, von, C. et al. Modeling initiation of Ewing sarcoma in human neural crest cells. PLoS ONE 6, e19305 (2011).

36. Bonafont, J. et al. Clinically relevant correction of recessive dystrophic epidermolysis bullosa by dual sgRNA CRISPR/Cas9-mediated gene editing. Mol. Ther. 27, 986-998 (2019).

37. $\mathrm{Li}$, J. et al. Efficient inversions and duplications of mammalian regulatory DNA elements and gene clusters by CRISPR/Cas9. J. Mol. Cell Biol. 7, 284-298 (2015).

38. Fukuhara, H., Ino, Y. \& Todo, T. Oncolytic virus therapy: a new era of cancer treatment at dawn. Cancer Sci. 107, 1373-1379 (2016).

39. Abbasalipour, M. et al. Improvement of K562 cell line transduction by FBS mediated attachment to the cell culture plate. Biomed. Res. Int. 2019 , 9540702-9540706 (2019).

40. Dai, X., Theobard, R., Cheng, H., Xing, M. \& Zhang, J. Fusion genes: a promising tool combating against cancer. Biochim. Biophys. Acta Rev. Cancer 1869, 149-160 (2018).

41. Schram, A. M., Chang, M. T., Jonsson, P. \& Drilon, A. Fusions in solid tumours: diagnostic strategies, targeted therapy, and acquired resistance. Nat. Rev. Clin. Oncol. 14, 735-748 (2017).

42. Paquet, D. et al. Efficient introduction of specific homozygous and heterozygous mutations using CRISPR/Cas9. Nature 533, 125-129 (2016).

43. Di Maio, M. et al. Symptomatic toxicities experienced during anticancer treatment: agreement between patient and physician reporting in three randomized trials. J. Clin. Oncol. 33, 910-915 (2015)

44. Kaelin, W. G. Common pitfalls in preclinical cancer target validation. Nat. Rev. Cancer 17, 425-440 (2017).

45. Lino, C. A., Harper, J. C., Carney, J. P. \& Timlin, J. A. Delivering CRISPR: a review of the challenges and approaches. Drug Deliv. 25, 1234-1257 (2018).

46. Horwood, N. J. et al. High-efficiency gene transfer into nontransformed cells: utility for studying gene regulation and analysis of potential therapeutic targets. Arthritis Res. 4, S215-S225 (2002).

47. Holkers, M. et al. Adenoviral vector DNA for accurate genome editing with engineered nucleases. Nat. Methods 11, 1051-1057 (2014).

48. Xu, C. L., Ruan, M. Z. C., Mahajan, V. B. \& Tsang, S. H. Viral Delivery Systems for CRISPR. Viruses 11, 28 (2019).

49. Lopes, A. et al. Oncolytic adenovirus drives specific immune response generated by a poly-epitope pDNA vaccine encoding melanoma neoantigens into the tumor site. J. Immunother. Cancer 7, 174 (2019).

50. Shaw, A. R. \& Suzuki, M. Immunology of adenoviral vectors in cancer therapy. Mol. Ther. Methods Clin. Dev. 15, 418-429 (2019).
51. Lee, C. S. et al. Adenovirus-mediated gene delivery: potential applications for gene and cell-based therapies in the new era of personalized medicine. Genes Dis. 4, 43-63 (2017).

52. Martínez-Vélez, N. et al. The oncolytic virus Delta-24-RGD elicits an antitumor effect in pediatric glioma and DIPG mouse models. Nat. Commun. 10, 2235-10 (2019).

53. Doench, J. G. et al. Optimized sgRNA design to maximize activity and minimize off-target effects of CRISPR-Cas9. Nat. Biotechnol. 34, 184-191 (2016).

54. Román-Rodríguez, F. J. et al. NHEJ-mediated repair of CRISPR-Cas9-induced DNA breaks efficiently corrects mutations in HSPCs from patients with fanconi anemia. Cell Stem Cell 25, 607-621.e7 (2019).

55. Muñoz-López, A. et al. Development refractoriness of MLL-rearranged human B cell acute leukemias to reprogramming into pluripotency. Stem Cell Rep. 7, 602-618 (2016).

56. Rodriguez-Perales, $\mathrm{S}$. et al. Truncated RUNX1 protein generated by a novel t $(1 ; 21)(\mathrm{p} 32 ; \mathrm{q} 22)$ chromosomal translocation impairs the proliferation and differentiation of human hematopoietic progenitors. Oncogene 35, 125-134 (2016).

57. Torres, R., García, A., Payá, M. \& Ramirez, J. C. Non-integrative lentivirus drives high-frequency cre-mediated cassette exchange in human cells. PLoS ONE 6, e19794 (2011).

58. Torres, R. \& Ramirez, J. C. A chemokine targets the nucleus: Cxcl12-gamma isoform localizes to the nucleolus in adult mouse heart. PLOS ONE 4, e7570 (2009).

59. Bae, S., Park, J. \& Kim, J.-S. Cas-OFFinder: a fast and versatile algorithm that searches for potential off-target sites of Cas9 RNA-guided endonucleases. Bioinformatics 30, 1473-1475 (2014).

60. Park, J., Lim, K., Kim, J.-S. \& Bae, S. Cas-analyzer: an online tool for assessing genome editing results using NGS data. Bioinformatics 33, 286-288 (2017).

\section{Acknowledgements}

This work was supported by grants from the Spanish National Research and Development Plan, Instituto de Salud Carlos III, and FEDER (PI17/02303 and DTS19/00111 to S.R-P.); AEI/MICIU EXPLORA Project BIO2017-91272-EXP and CaixaImpulse (CI1800017;FuGe) to S.R-P. RT-R. is supported by a postdoctoral fellowship from the Asociación Española Contra el Cáncer (AECC). P.M. is supported by the European Research Council (CoG-2014-646903 and PoC-2018-811220), the Spanish Ministry of Science, Innovation and Universities (SAF2016), and the Catalunya Government (SGR330 and PERIS 2017). J.C.S. is supported by the Spanish Ministry of Science, Innovation and Universities (SAF2017-84248-P) and the Spanish Cell Therapy cooperative research network (TERCEL)(RD16/0011/0011). C.B. is supported by the AECC, Beca FERO, and the ISCIII/FEDER (PI17/01028). P.M. also acknowledges the financial support from the Obra Social La Caixa-Fundaciò Josep Carreras. P.M. is an investigator of the Spanish Cell Therapy cooperative research network (TERCEL). A.M.C. acknowledges funding from ISCIII-FEDER (CP13/00189) and Xarxa de Bancs de Tumors de Catalunya (XBTC; sponsored by Pla Director d'Oncologia de Catalunya). We thank Dr Kenneth McCreath for critical reading of the paper.

\section{Author contributions}

R.T-R. and S.R-P. conceived the project, designed and performed experiments, analyzed the data and wrote the manuscript. M.M-L., did the experiments, analyzed results, and wrote the paper. P.M-G., P.P-S.; O.Q-B.; S.G-S.; P.P.; M.C.M. and F.J.M. assisted in the experiments and collected data. C.B.; H.P.; J.C.S. and P.M. analyzed the data and commented on the paper. All authors read and approved the final manuscript. A.M.C. and J.M. provide the PDX tumors and commented the paper.

\section{Competing interests}

A patent has been filed relating to the data presented in this research study by S.R-P., R.T-R. and M.M-L. (EP18382746.8). The remaining authors declare no competing interests.

\section{Additional information}

Supplementary information is available for this paper at https://doi.org/10.1038/s41467020-18875-x.

Correspondence and requests for materials should be addressed to R.T.-R. or S.R.-P.

Peer review information Nature Communications thanks the anonymous reviewer(s) for their contribution to the peer review of this work. Peer reviewer reports are available.

Reprints and permission information is available at http://www.nature.com/reprints

Publisher's note Springer Nature remains neutral with regard to jurisdictional claims in published maps and institutional affiliations. 
(c) (i) Open Access This article is licensed under a Creative Commons Attribution 4.0 International License, which permits use, sharing, adaptation, distribution and reproduction in any medium or format, as long as you give appropriate credit to the original author(s) and the source, provide a link to the Creative Commons license, and indicate if changes were made. The images or other third party material in this article are included in the article's Creative Commons license, unless indicated otherwise in a credit line to the material. If material is not included in the article's Creative Commons license and your intended use is not permitted by statutory regulation or exceeds the permitted use, you will need to obtain permission directly from the copyright holder. To view a copy of this license, visit http://creativecommons.org/ licenses/by/4.0/.

(C) The Author(s) 2020 